TRANSACTIONS OF THE

AMERICAN MATHEMATICAL SOCIETY

Volume 351, Number 10, Pages 4205-4243

S 0002-9947(99)02461-7

Article electronically published on June 10, 1999

\title{
WEIGHTED LAPLACE TRANSFORMS AND BESSEL FUNCTIONS ON HERMITIAN SYMMETRIC SPACES
}

\author{
HONGMING DING
}

\begin{abstract}
This paper defines $\pi$-weighted Laplace transforms on the spaces of $\pi$-covariant functions. By the inverse Laplace transform we define operatorvalued Bessel functions. We also study the holomorphic discrete series of the automorphism group of a Siegel domain of type II.
\end{abstract}

\section{INTRODUCTION}

In this paper, we develop the theory of operator-valued Bessel functions on general Siegel domains, and apply these Bessel functions to study representations of their automorphism groups. The significance of this work can be described roughly as follows.

Bounded symmetric domains form a class of domains in several complex variables that generalize the ordinary unit disk in one variable. These domains are classified Lie theoretically as Hermitian symmetric spaces $G / K$ having complex structure. For most of these domains the reductive Lie group $G$ is a classical group, but in some cases the group $G$ is "exceptional." Moreover, certain of these domains are of "tube type" - that is, can be realized as half spaces in $\mathbb{C}^{n}$, while others are not. In a previous paper with K. Gross [DG1] we developed the theory of operatorvalued Bessel functions for Hermitian symmetric spaces of tube type. We now complete that work by placing the theory in the general setting of an arbitrary Hermitian symmetric space, whether or not it is of tube type. Thus, the results in this paper provide a general construction, independent of domain by domain analysis, that includes not only the classical bounded symmetric domains but also the exceptional ones as well. In our work, it is convenient to frame our theory in terms of the unbounded realization of $G / K$ as a generalized Siegel domain, realized in terms of the structure of Jordan pairs. For background on Jordan pairs, we refer to Section 2 below and [L1].

We now outline the main ideas in this paper.

Let $\left(V, V^{-}\right)$be a simple Jordan pair with a positive Hermitian involution. With respect to a maximal tripotent $e, V$ is decomposed as $V=V_{2} \oplus V_{1}$. Then $V_{2}$ is the complexification of a formally real Jordan algebra $J$, in which the interior

Received by the editors March 22, 1994 and, in revised form, January 9, 1997.

1991 Mathematics Subject Classification. Primary 22E46, 43A85, 17C30, 33C10; Secondary 22E30, 17C50, 33B15.

Key words and phrases. $\pi$-weighted Laplace transform, Jordan pair, Siegel domain of type II, Bessel function, holomorphic discrete series.

This research was supported in part by the National Science Foundation grant DMS-9312465. 
$\Omega$ of sets of squares is the positive cone. With a positive definite bilinear map $B: V_{1} \times V_{1}^{-} \rightarrow V_{2}$ we can define a Siegel domain $\Sigma$ of type II by

$$
\Sigma=\left\{x_{2}+x_{1} \in V_{2} \oplus V_{1} \mid \operatorname{Im} x_{2}-\frac{1}{2} B\left(x_{1}, \bar{x}_{1}\right)>0\right\} .
$$

The Cayley transform $c$ is a biholomorphic isomorphism of a circled bounded symmetric domain $D$ with $\Sigma$.

Let $G^{\prime}$ and $G$ be the groups of biholomorphic automorphisms of $D$ and $\Sigma$, respectively, let $K$ be the maximal compact subgroup of $G^{\prime}$, and $K^{\mathbb{C}}$ the complexification of $K$. We decompose the Lie algebra $\mathfrak{g}$ of $G$ as a canonical graduation

$$
\mathfrak{g}=\mathfrak{g}_{-1} \oplus \mathfrak{g}_{-\frac{1}{2}} \oplus \mathfrak{g}_{0} \oplus \mathfrak{g}_{\frac{1}{2}} \oplus \mathfrak{g}_{1},
$$

and denote by $G_{-1}, G_{-\frac{1}{2}}, G_{0}, G_{\frac{1}{2}}$, and $G_{1}$ the corresponding analytic subgroups. Let $A$ be the connected component of the identity in $G_{0}$, and let $L$ be the maximal compact subgroup of $A$. We identify $V_{1}$ with a subgroup $K_{-\frac{1}{2}}$ of $K^{\mathbb{C}}$ by the map $u_{0} \mapsto k\left(u_{0}\right)$. Note that as subgroups of $K^{\mathbb{C}}, A$ normalizes $K_{-\frac{1}{2}}$ (cf. (4.8.2)), and $A K_{-\frac{1}{2}}$ is a subgroup of $K^{\mathbb{C}}$.

Let $\pi$ be a finite-dimensional irreducible holomorphic representation of $K^{\mathbb{C}}$, denote by $\mathcal{V}=\mathcal{V}_{\pi}$ the space on which $\pi$ acts and by $\mathcal{E}=\mathcal{E}_{\pi}$ the space of all linear transformations on $\mathcal{V}$. We will study $\mathcal{V}$-valued or $\mathcal{E}$-valued measurable functions $f$ on $A K_{-\frac{1}{2}}$, which are holomorphic in $u_{0} \in V_{1} . f$ is called $\pi$-covariant if

$$
f\left(a k\left(u_{0}\right) l\right)=\pi(l)^{-1} f\left(a k\left(u_{0}\right)\right)
$$

for all $a \in A, u_{0} \in V_{1}$, and $l \in L$. Let $\Delta$ be the Koecher norm function and $\mu$ be a constant given in Section 3.4. Whenever the integral

$$
\begin{aligned}
F(z, u)=\pi^{-\frac{n}{2}} & \int_{A K_{-\frac{1}{2}}} e^{i(z \mid a e)} e^{\left(u \mid a u_{0}\right)} e^{-\left(u_{0} \mid u_{0}\right)} \\
& \times \pi\left(a k\left(i u_{0}\right)\right)^{*-1} f\left(a k\left(u_{0}\right)\right) \Delta(a e)^{\mu} d a d u_{0}
\end{aligned}
$$

is absolutely convergent for all $(z, u) \in \Sigma$, the function $F$ on $\Sigma$ is called the $\pi$ weighted Laplace transform of $f$.

The operator-valued Bessel function of weight $\pi$ is the function $K_{\pi}:\left(A K_{-\frac{1}{2}}\right) \times$ $V_{1} \rightarrow \mathcal{E}$ obtained by Laplace inversion from the formula

$$
\begin{aligned}
& \int_{A K_{-\frac{1}{2}}} e^{i(z \mid a e)} e^{\left(u \mid a u_{0}\right)} e^{-\left(u_{0} \mid u_{0}\right)} \pi\left(a k\left(i u_{0}\right)\right)^{*-1} K_{\pi}\left(a k\left(u_{0}\right) ; v_{0}\right) d a d u_{0} \\
& =e^{-i\left(z^{-1} \mid e\right)} e^{\left(z^{-1} \mid B\left(v_{0}, \bar{u}\right)\right)} \pi(z) \pi(k(u)) \pi\left(k\left(-i v_{0}\right)\right)^{*}
\end{aligned}
$$

for all $(z, u) \in \Sigma$ and $v_{0} \in V_{1}$, where $\pi(z)$ is given by (4.2.1). Let $M=$ $\left(m_{1}, m_{2}, \cdots, m_{r}\right)$ with $m_{1} \geq m_{2} \geq \cdots \geq m_{r}$ be the signature of the restricted lowest weight of $\pi$, and set $\omega(\pi)=m_{r}$. In this paper, we assume

$$
\omega(\pi)>2 \mu+b-1
$$

where $b$ is given in Section 3.3. In this range, Laplace transform of (5) is valid, and the Bessel functions $K_{\pi}$ also satisfy the square-integrability

$$
\int_{\left(A K_{-\frac{1}{2}}\right) \times V_{1}}\left\|K_{\pi}\left(a k(u) ; v_{0}\right)\right\|^{2} \Delta(a e)^{-\mu-b} e^{-(u \mid u)} e^{-\left(v_{0} \mid v_{0}\right)} d a d u d v_{0}<\infty .
$$


Let $\mathfrak{S F}_{\pi}\left(A K_{-\frac{1}{2}}\right)$ be the space of all $\pi$-covariant measurable functions $f: A K_{-\frac{1}{2}}$ $\rightarrow \mathcal{V}_{\pi}$, which are holomorphic in $u_{0}$ and

$$
\int_{A K_{-\frac{1}{2}}}\left\|f\left(a k\left(u_{0}\right)\right)\right\|^{2} e^{-\left(u_{0} \mid u_{0}\right)} d a d u_{0}<\infty .
$$

$\mathfrak{S F}_{\pi}\left(A K_{-\frac{1}{2}}\right)$ is a Hilbert space with respect to an inner product, and we denote the resulting Hilbert space by $\mathfrak{S F}_{\Gamma}$. Using the Bessel function $K_{\pi}$, we define the Hankel transform $\mathcal{K}_{\pi}$ on $\mathfrak{S F}_{\Gamma}$ by

$$
\left(\mathcal{K}_{\pi} f\right)\left(a k\left(u_{0}\right)\right)=\int_{A K_{-\frac{1}{2}}} K_{\pi}\left(a_{0}^{*} a k\left(u_{0}\right) ; v_{0}\right) f\left(a k\left(v_{0}\right)\right) e^{-\left(v_{0} \mid v_{0}\right)} d a_{0} d v_{0} .
$$

We construct a unitary operator from $\mathfrak{S F}_{\Gamma}$ to a Hilbert space $\mathcal{H}_{\pi}$ of holomorphic $\mathcal{V}$-valued functions on $\Sigma$ which intertwines two representations $S_{\pi}$ and $T_{\pi}$ of $G$ indexed by finite-dimensional irreducible holomorphic representations $\pi$ of $K^{\mathbb{C}}$. Denote by $j$ the inversion element $(z, u) \mapsto\left(-z^{-1}, z^{-1} \circ u\right)$ of $G$. Then the operator $S_{\pi}(j)$ is the Hankel transform $\mathcal{K}_{\pi}$, and the square-integrability (7) of the Bessel functions $K_{\pi}$ implies the square-integrability of $S_{\pi}$. We show also the irreducibility of the representations $T_{\pi}$ (and of $S_{\pi}$ ). Then $S_{\pi}$ (and also $T_{\pi}$ ) are in the holomorphic discrete series of $G$.

The paper is organized as follows.

In Section 2, we review the definitions, structures and properties of Jordan pairs and associated symmetric spaces. In Section 3, we study the automorphism group $G$ of a Siegel domain $\Sigma$ of type II, generating elements and the multipliers of $G$. Section 4 defines the operator-valued gamma function and the $\pi$-weighted Laplace transform. In Section 5, we define the operator-valued Bessel functions and study their square-integrability. In Section 6 , we introduce the unitary equivalent Hilbert spaces $\mathfrak{S F}_{\Gamma}$ and $\mathcal{H}_{\pi}$, where $\mathcal{H}_{\pi}$ are reproducing kernel spaces. We define the Hankel transforms by Bessel functions. In Section 7 , we construct unitary representations $S_{\pi}$ and $T_{\pi}$ of $G$ on $\mathfrak{S F}_{\Gamma}$ and $\mathcal{H}_{\pi}$. We prove the irreducibility and the squareintegrability of $S_{\pi}$ and $T_{\pi}$. We compute the reproducing kernel of $\mathcal{H}_{\pi}$ explicitly.

We turn to the relationship of this paper to other literature.

In last decades, a number of mathematicians (cf. [B1], [Her], [Gi], [J], [GR], [Dib]) investigated Bessel functions on matrix spaces, and more generally, on symmetric cones. In [GK1] and [GK2], two kinds of operator-valued Bessel functions on matrix spaces were studied. In [DG1], we generalized the second or the socalled reduced Bessel functions from matrix spaces to symmetric cones in general by the Jordan algebra structure, and applied these functions to study representations of automorphism groups of all Hermitian symmetric spaces of tube type. In [Di1]-[Di3], we developed a theory of operator-valued Bessel functions on mixed Schrödinger-Fock spaces, and studied the harmonic representations and holomorphic discrete series for the groups $U(p, q)$ and $O_{*}(2 m)$ with $m$ odd. These groups are known as automorphism groups of certain Hermitian symmetric spaces, which are not of tube type and are called Siegel domains of type II.

Upon these stepping stones, we now broaden the scope of Bessel function theory to all Siegel domains by the structure of Jordan pairs and the theory of Siegel domains, which were developed by a number of authors (cf. [L1]-[L3], [Do1], [Do2], $[\mathrm{Gi}],[\mathrm{Kan}],[\mathrm{M}],[\mathrm{P}])$. We study also representations of the automorphism group of $\Sigma$. 
As related works, the harmonic representation of certain groups was studied in $[\mathrm{Sh}],[\mathrm{Se}]$ and $[\mathrm{We}]$. [KV] studied the harmonic representation of $U(p, q)$ by pluriharmonic polynomials. [Ho1], [Ho2] developed the theory of dual reductive pairs.

In the next paper [DG2], we will consider the analytic continuation of the Bessel function and analytic continuation of the holomorphic discrete series for the universal covering group of $G$, as well as other spaces in the Wallach set (cf. [W1], [W2], [EHW]) associated to repesentations $\pi$. We will also study the operator-valued hypergeometric functions on Hermitian symmetric spaces.

I wish to thank Professors J. Dorfmeister, K. Gross and O. Loos for their encouragement and helpful discussions.

\section{JORDAN PAIRS AND SYMMETRIC SPACES}

In this section, we review briefly the definitions and structures of Jordan pairs and associated symmetric spaces, and list the properties that are needed in this paper. We refer to [L1] and [FK2] for more detail.

2.1. Jordan pairs. Let $V^{+}$and $V^{-}$be complex vector spaces, and let $Q_{+}$: $V^{+} \rightarrow \operatorname{Hom}\left(V^{-}, V^{+}\right)$and $Q_{-}: V^{-} \rightarrow \operatorname{Hom}\left(V^{+}, V^{-}\right)$be quadratic maps; i.e., $Q_{+}(\alpha v)=\alpha^{2} Q_{+}(v)$ and $Q_{-}\left(\alpha v^{-}\right)=\alpha^{2} Q_{-}\left(v^{-}\right)$for $\alpha \in \mathbb{C}, v \in V^{+}$and $v^{-} \in V^{-}$. Define trilinear maps \{\}$: V^{\sigma} \times V^{-\sigma} \times V^{\sigma} \rightarrow V^{\sigma}$ and bilinear maps $D_{\sigma}: V^{\sigma} \times$ $V^{-\sigma} \rightarrow \operatorname{End}\left(V^{\sigma}\right)$ by

$$
\{x y z\}=D_{\sigma}(x, y) z=Q_{\sigma}(x+z) y-Q_{\sigma}(x) y-Q_{\sigma}(z) y
$$

for $x, z \in V^{\sigma}, y \in V^{-\sigma},(\sigma= \pm)$. The pair $\left(V^{+}, V^{-}\right)$together with the quadratic maps $\left(Q_{+}, Q_{-}\right)$is called a Jordan pair if the identity

$$
\{u v\{x y z\}\}-\{x y\{u v z\}\}=\{\{u v x\} y z\}-\{x\{v u y\} z\}
$$

holds for all $u, x, z \in V^{\sigma}, v, y \in V^{-\sigma}, \sigma= \pm$.

A Hermitian involution of a complex Jordan pair is an invertible complex antilinear map $\delta: V^{+} \rightarrow V^{-}$such that $\delta\left(Q_{+}(x) \delta y\right)=Q_{-}(\delta x) y$ for all $x, y \in V^{+}$. We say $\delta$ is positive if $Q_{+}(x) \delta x=\lambda x$ for $\lambda \in \mathbb{C}$ implies $\lambda>0$ for all non-zero $x \in V^{+}$.

2.2. Jordan algebras. A (linear) Jordan algebra is a vector space $J$ having a unit $e$ with a commutative bilinear multiplication $x \circ y$ satisfying $x^{2} \circ(x \circ y)=x \circ\left(x^{2} \circ y\right)$, where $x^{2}=x \circ x$. The quadratic representation $P$ of $J$ is defined by

$$
P(x) y=\frac{1}{2} x \circ(x \circ y)-\frac{1}{4} x^{2} \circ y
$$

for all $x, y \in J$. (Note: Here, the product $x \circ y$ is twice of what it is in some literature. By this assumption, (2.7.5) below is simpler.) The representation $P$ of $J$ can be extended to its complexification $J^{\mathbb{C}} . z \in J^{\mathbb{C}}$ is invertible if and only if $P(z)$ is invertible and then

$$
P\left(z^{-1}\right)=P(z)^{-1}
$$

By [Koe], VII.1(7),

$$
P\left(z^{*}\right)[P(\operatorname{Im} z)]^{-1} P(z)=\left[P\left(\operatorname{Im}\left(-z^{-1}\right)\right)\right]^{-1}
$$

and

$$
P(z)[P(\operatorname{Im} z)]^{-1} P\left(z^{*}\right)=\left[P\left(\operatorname{Im}\left(-z^{-1}\right)\right)\right]^{-1}
$$


for invertible $z \in J^{\mathbb{C}}$, where $z^{*}$ is the complex conjugate of $z$ in $J^{\mathbb{C}}$ and $\operatorname{Im} z=$ $\frac{1}{2 i}\left(z-z^{*}\right)$. A real Jordan algebra is formally real if $x^{2}+y^{2}=0$ implies $x=y=0$. Every Jordan algebra $J$ gives rise to a Jordan pair by setting $V^{+}=V^{-}=J$ and $Q_{+}(x) y=Q_{-}(x) y=P(x) y$ for $x, y \in J$.

A non-empty, open, self-dual, homogeneous cone in a finite-dimensional Euclidean space is called a symmetric cone. In a formally real Jordan algebra $J$ the interior $\Omega$ of the set $\left\{x^{2} \mid x \in J\right\}$ of all squares is a symmetric cone, and any symmetric cone is isomorphic to a cone arising from a formally real Jordan algebra in this way. The elements of $\Omega$ are invertible in $J$, and $\Omega$ is the connected component of $e$ in the set of invertible elements of $J$.

2.3. Tripotents. To simplify the notation, we omit the subscript and superscript + ; i.e., we write $V, Q, D$ for $V^{+}, Q_{+}, D_{+}$, respectively. An idempotent in a Jordan pair $\left(V, V^{-}\right)$is a pair $(a, b) \in V \times V^{-}$such that $Q(a) b=a$ and $Q_{-}(b) a=b$. If $\left(V, V^{-}\right)$has a Hermitian involution $\delta$ and denotes $\delta v=\bar{v}$, then the set of all real points of the idempotent set is the set of all idempotents of the form $(e, \bar{e})$ and thus may be identified (by projection onto the first factor) with the set $M$ of $e$ in $V$ satisfying $Q(e) \bar{e}=e$. Such an element $e \in V$ is called a tripotent; i.e., $e$ is called a tripotent if $Q(e) \bar{e}=e$.

2.4. Peirce decomposition. Let $\left(V, V^{-}\right)$be a Jordan pair with the Hermitian involution $\delta, \delta x=\bar{x}$, and let $e$ be a tripotent of $V$. Then we have a Peirce decompositon of $V$ with respect to $e$.

(a) $V$ decomposes as

$$
V=V_{2} \oplus V_{1} \oplus V_{0}
$$

where $V_{\alpha}=V_{\alpha}(e)$ is the $\alpha$-eigenspace of $D(e, \bar{e})$. The $V_{\alpha}$ are orthogonal with respect to any associative inner product and satisfy the multiplication rules

$$
\left\{V_{\alpha} \bar{V}_{\beta} V_{\gamma}\right\} \subset V_{\alpha-\beta+\gamma}, \quad\left\{V_{2} \bar{V}_{0} V\right\}=\left\{V_{0} \bar{V}_{2} V\right\}=0 .
$$

In particular, $\left(V_{\alpha}, \bar{V}_{\alpha}\right)$ is a $\delta$-invariant subpair for $\alpha=0,1,2$.

(b) $V_{2}$ is a complex Jordan algebra with Jordan product $x \circ y=\{x \bar{e} y\}$ and the unit element $e$. The map $z \mapsto z^{*}=Q(e) \bar{z}$ is a complex antilinear automorphism of period 2 of the Jordan algebra $V_{2}$.

(c) The fixed point set $J=J(e)$ of the map $z \mapsto z^{*}$ is a real Jordan algebra, and $V_{2}=J \oplus i J$ is the complexification of $J$. If $\delta$ is positive, then $J$ is formally real.

2.5. Circled bounded symmetric domains. Let $D$ be a bounded domain in a finite-dimensional complex vector space $V . D$ is called symmetric if, for every $z \in D$, there exists an automorphism $S_{z}$ of period two of $D$, having $z$ as an isolated fixed point. $D$ is called circled (with respect to 0 ) if $0 \in D$, and $e^{i t} z \in D$ for all $z \in D, t \in \mathbb{R}$.

Every bounded symmetric domain in $V$ is isomorphic to a bounded symmetric and circled domain which is unique up to a linear isomorphism of $V$.

Let $\left(V, V^{-}\right)$be a Jordan pair with a positive Hermitian involution. Then every $v \in V$ can be written uniquely as

$$
v=\lambda_{1} e_{1}+\lambda_{2} e_{2}+\cdots+\lambda_{n} e_{n}
$$


Here the $e_{i}$ are pairwise orthogonal non-zero tripotents which are real combinations of powers of $v$, and the $\lambda_{i}$ satisfy

$$
0 \leq \lambda_{1} \leq \lambda_{2} \leq \cdots \leq \lambda_{n}
$$

We call (1) the spectral decomposition of $v$ and the $\lambda_{i}$ the eigenvalues of $v$.

Let $D$ be a circled bounded symmetric domain. Then $D$ is the open unit ball of the spectral norm of the associated Jordan pair $\left(V, V^{-}\right)$with the positive Hermitian involution $\delta: v \mapsto \bar{v}$, where the spectral norm $|v|$ of $v \in V$ denotes the largest eigenvalue of $v$. Conversely, given a Jordan pair with the positive Hermitian involution, the open unit ball of the spectral norm is a circled bounded symmetric domain whose associated Jordan pair is the given one.

2.6. Simple Jordan pairs. A Jordan pair $\left(V, V^{-}\right)$is called simple if the $Q$ operators are non-trivial, and if it contains no proper ideals. Here an ideal of $\left(V, V^{-}\right)$is a pair $\left(I, I^{-}\right)$of subspaces such that $\left\{I V^{-} V\right\}+\left\{V I^{-} V\right\} \subset I$ and $\left\{I^{-} V V^{-}\right\}+\left\{V^{-} I V^{-}\right\} \subset I^{-}$. A finite-dimensional semisimple Jordan pair is the direct sum of simple ideals which are unique up to order.

The corresponding concept for domains is irreducibility. A circled bounded symmetric domain $D$ is called irreducible if it is not isomorphic to a direct product $D^{\prime} \times D^{\prime \prime}$ of lower-dimensional circled bounded symmetric domains. The domain $D$ is irreducible if and only if $\left(V, V^{-}\right)$is simple. The classification of simple Jordan pairs with positive involutions can be found in [L1].

2.7. Siegel domains of type II. As in Section 2.5, let $D$ be a circled bounded symmetric domain, $G^{\prime}$ its automorphism group, $K$ the isotropy subgroup of 0 in $G^{\prime}$, and let $(\cdot \mid \cdot)$ be a $K$-invariant Hermitian inner product on $V$. We denote by $M$ the set of all tripotents of $V$, and define an ordering on $M$ by

$$
c<e \Longleftrightarrow e-c \in M \text { and } c \perp(e-c) \text {. }
$$

A tripotent $e$ is maximal with respect to this ordering if and only if the Peirce space $V_{0}(e)=0$.

Let $(V, \bar{V})$ be a Jordan pair with the Hermitian involution $v \mapsto \bar{v}$, and $e$ be a maximal tripotent of $V$. Since $V_{0}(e)=0, x=x_{2}+x_{1}$ is the decomposition of $x \in D$ in the Peirce spaces, where $x_{2} \in V_{2}(e)$ and $x_{1} \in V_{1}(e)$. Let

$$
\Sigma=\left\{x_{2}+x_{1} \in V_{2} \oplus V_{1} \mid \operatorname{Im} x_{2}-\frac{1}{2} B\left(x_{1}, \bar{x}_{1}\right)>0\right\},
$$

where $\operatorname{Im} x_{2}=\frac{1}{2 i}\left(x_{2}-x_{2}^{*}\right)$ and the bilinear map $B: V_{1} \times \bar{V}_{1} \rightarrow V_{2}$ is defined by

$$
B(u, \bar{v})=\{u \bar{v} e\} \quad\left(u \in V_{1}, \bar{v} \in \bar{V}_{1}\right) .
$$

$\Sigma$ is called a Siegel domain of type II. Note that B is Hermitian and positive definite in the sense that

$$
\begin{gathered}
B(u, \bar{v})^{*}=B(v, \bar{u}), \\
B(u, \bar{u}) \geq 0,
\end{gathered}
$$

and $B(u, \bar{u})=0$ only if $u=0$. For $z \in V_{2}, u \in V_{1}$, define

$$
z \circ u=\{z \bar{e} u\} .
$$


Let $x=x_{2}+x_{1} \in V_{2} \oplus V_{1}$. The Cayley transform $c$ given by

$$
c(x)=\frac{1}{2}\left(x_{2}+i e\right) \circ\left(i x_{2}+e\right)^{-1}+\sqrt{2}\left(i x_{2}+e\right)^{-1} \circ x_{1}
$$

is a biholomorphic isomorphism of $D$ with $\Sigma$.

By [L1], Proposition 10.11, we have

$$
P(z) B(u, \bar{v})=B\left(z \circ u, \overline{z^{*} \circ v}\right),
$$

and

$$
z \circ B(u, \bar{v})=B(z \circ u, \bar{v})+B\left(u, \overline{z^{*} \circ v}\right)
$$

for all $z \in V_{2}, u, v \in V_{1}$.

By [Do1], (2.7),

$$
(P(z) w) \circ u=z \circ(w \circ(z \circ u))
$$

for all $z, w \in V_{2}, u \in V_{1}$. By (2.2.3), (2.2.4) and (4), we have

$$
z \circ\left((\operatorname{Im} z)^{-1} \circ\left(z^{*} \circ u\right)\right)=\operatorname{Im}\left(-z^{-1}\right) \circ u
$$

and

$$
z^{*} \circ\left((\operatorname{Im} z)^{-1} \circ(z \circ u)\right)=\operatorname{Im}\left(-z^{-1}\right) \circ u
$$

for all $u \in V_{1}$ and invertible $z \in V_{2}$.

2.8. Remark. In this paper, we study the circled bounded symmetric domain $D$ and the Siegel domain $\Sigma$ of type II associated with a simple Jordan pair $\left(V, V^{-}\right)$. $\left(V, V^{-}\right)$has a positive Hermitian involution $v \mapsto \bar{v}$. $e$ is a maximal tripotent of $V, V_{0}(e)=0$ and $V=V_{2} \oplus V_{1}$. Then $V_{2}$ is the complexification of a formally real Jordan algebra $J$, in which the interior $\Omega$ of sets of squares is the positive cone, $e$ is the unit element of $J$, and $(\cdot \mid \cdot)$ is a $K$-invariant inner product on $V$ (in particular, on $V_{2}$ ). We assume that $\Omega$ is an irreducible symmetric cone in the simple Jordan algebra $J$.

\section{Automorphism groups and Lie Algebra structures}

In this section, we recall the properties of automorphism groups of circled bounded symmetric domains $D$ and Siegel domains $\Sigma$ of type II, their Lie algebras and complexifications, which are important for the sequel. We refer to [FK1], [RV1], and [Do1] for more detail. We also study generating elements and multipliers for the automorphism group of $\Sigma$.

3.1. Automorphism group of $D$. Let $G^{\prime}$ be the automorphism group of $D$ and $\mathfrak{g}^{\prime}$ its Lie algebra. Let us consider the Cartan decomposition $\mathfrak{g}^{\prime}=\mathfrak{k}+\mathfrak{p}$, where $\mathfrak{k}$ has non-trivial center and $\mathfrak{h}$ will be a Cartan subalgebra of $\mathfrak{k}$ (and hence also of $\mathfrak{g}^{\prime}$ ). $\mathfrak{g}^{\mathbb{C}}, \mathfrak{k}^{\mathbb{C}}, \mathfrak{p}^{\mathbb{C}}, \mathfrak{h}^{\mathbb{C}}$ are their complexifications, respectively.

The $\mathfrak{h}^{\mathbb{C}}$-roots of $\mathfrak{g}^{\mathbb{C}}$ that are also roots of $\mathfrak{k}^{\mathbb{C}}$ are called compact roots. We denote by $\Phi^{+}$the set of positive non-compact roots. Denoting by $\tau$ the conjugation with respect to the real form $\mathfrak{k}+i \mathfrak{p}$, we consider a basis of root vectors $e_{\alpha}$ such that $\tau e_{\alpha}=-e_{-\alpha},\left[e_{\alpha}, e_{-\alpha}\right]=h_{\alpha},\left[h_{\alpha}, e_{ \pm \alpha}\right]= \pm 2 e_{ \pm \alpha}$. Setting

$$
\mathfrak{p}^{ \pm}=\sum_{\alpha \in \Phi^{+}} \mathbb{C} e_{ \pm \alpha},
$$


we have

$$
\mathfrak{g}^{\mathbb{C}}=\mathfrak{p}^{-}+\mathfrak{k}^{\mathbb{C}}+\mathfrak{p}^{+} .
$$

On $\mathfrak{p}^{+}$we have a Hermitian inner product defined by

$$
(z \mid w)=-K(z, \tau w)
$$

where $K$ is the Killing form.

$\gamma_{1}, \gamma_{2}, \cdots, \gamma_{r}$ will be strongly orthogonal roots of Harish-Chandra, with the ordering $\gamma_{1}>\gamma_{2}>\cdots>\gamma_{r}$. $r$ is the real rank of $\mathfrak{g}^{\prime}$. We will use the abbreviated notations

$$
e_{j}=e_{\gamma_{j}}, \quad h_{j}=h_{\gamma_{j}} \quad(1 \leq j \leq r), \quad e=\sum_{1}^{r} e_{j} .
$$

We denote by $\mathfrak{h}^{-}$the real span of vectors $i h_{j}$, by $\mathfrak{a}^{+}$the real span of the $e_{j}$, and by $\mathfrak{a}$ the real span of the vectors $i\left(e_{j}+\tau e_{j}\right),(1 \leq j \leq r)$. $\mathfrak{a}$ is a Cartan subalgebra of the pair $\left(\mathfrak{g}^{\prime}, \mathfrak{k}\right)$.

$G^{\mathbb{C}}$ will be the adjoint group of $\mathfrak{g}^{\mathbb{C}} . G^{\prime}, K, K^{\mathbb{C}}, P^{ \pm}, A$ will be analytic subgroups corresponding to $\mathfrak{g}^{\prime}, \mathfrak{k}, \mathfrak{k}^{\mathbb{C}}, \mathfrak{p}^{ \pm}, \mathfrak{a}$. We identify $\mathfrak{p}^{+}$with $P^{+}$by the exponential map.

3.2. Cayley transform. Let $c$ be the Cayley transform given by (2.7.3). We write $G$ for $c G^{\prime} c^{-1}$ and $\mathfrak{g}$ for its Lie algebra. Let $\mathfrak{g}_{T}^{\prime}$ be the fixed point set of $\mathfrak{g}^{\prime}$ under $A d\left(c^{4}\right)$ and $\mathfrak{g}_{T}^{\mathbb{C}}$ its complexification. The intersections of $\mathfrak{k}, \mathfrak{p}, \mathfrak{p}^{+}$with $\mathfrak{g}_{T}^{\mathbb{C}}$ give corresponding decompositions $\mathfrak{g}_{T}^{\prime}=\mathfrak{k}_{T}+\mathfrak{p}_{1}$ and $\mathfrak{g}_{T}^{\mathbb{C}}=\mathfrak{p}_{1}^{-}+\mathfrak{k}_{T}^{\mathbb{C}}+\mathfrak{p}_{1}^{+}$.

$\mathfrak{n}_{1}^{ \pm}=\mathfrak{g} \cap \mathfrak{p}_{1}^{ \pm}$is a real form of $\mathfrak{p}_{1}^{ \pm} . A d\left(c^{2}\right)$ is a Cartan involution of $\mathfrak{k}_{T}$; the corresponding decomposition is $\mathfrak{k}_{T}=\mathfrak{l}_{T}+\mathfrak{q}_{1}$. Writing $\mathfrak{k}_{T}^{*}=\mathfrak{l}_{T}+i \mathfrak{q}_{1}, K_{T}^{*}$ and $L_{T}$ for the corresponding analytic groups of $\mathfrak{k}_{T}^{*}$ and $\mathfrak{l}_{T}$, and $L_{T}^{0}$ for the connected component of the identity in $L_{T}$, the orbit $\Omega=K_{T}^{*} \cdot e \cong K_{T}^{*} / L_{T}^{0}$ is a homogeneous selfdual cone in $\mathfrak{n}_{1}^{+}$.

$\mathfrak{n}_{1}^{+}$has the structure of a formally real Jordan algebra $J$, in which $\Omega$ is the interior of the set of squares, $e$ is a maximal tripotent of $V, \mathfrak{p}_{1}^{+}$coincides with $V_{2}$, $\mathfrak{p}^{+}$coincides with $V=V_{2} \oplus V_{1}$ (cf. Section 2.4), and the inner product defined by (3.1.2) coincides with one given in Section 2.7.

The Cayley transform $\mathfrak{g}_{T}$ of $\mathfrak{g}_{T}^{\prime}$ has the vector space decomposition

$$
\mathfrak{g}_{T}=\mathfrak{n}_{1}^{-}+\mathfrak{k}_{T}^{*}+\mathfrak{n}_{1}^{+} .
$$

We define $\mathfrak{m}$ to be the centralizer of $i \mathfrak{h}^{-}$in $\mathfrak{l}_{T}$; it is then also the centralizer of $i \mathfrak{h}^{-}$ in $\mathfrak{g}_{T}$ and $\mathfrak{g}$.

3.3. Restricted root space decompositions. We have $i \mathfrak{h}^{-} \subset i \mathfrak{q}_{1} \subset \mathfrak{g}_{T}$, and, since $A d(c)$ interchanges $i \mathfrak{h}^{-}$with $\mathfrak{a}, i \mathfrak{h}^{-}$is a Cartan subalgebra of the pair $(\mathfrak{g}, \mathfrak{k})$ as well as of $\left(\mathfrak{k}_{T}^{*}, \mathfrak{l}_{T}\right)$. The $i \mathfrak{h}^{-}$-roots of $\mathfrak{g}$ are $\pm \frac{1}{2}\left(\gamma_{j} \pm \gamma_{k}\right), \pm \gamma_{j}, \pm \frac{1}{2} \gamma_{j}, \quad(1 \leq j, k \leq r)$ with respective multiplicities $\nu, 1$ and $2 b$ (independent of $j, k$ ). The corresponding (restricted) root spaces refine the decompositions (3.2.1) and (3.1.1) as follows:

$$
\begin{gathered}
\mathfrak{n}_{1}^{+}=\sum_{j<k} \mathfrak{n}^{+j k}+\sum_{j} \mathfrak{n}^{+j} \\
\mathfrak{p}_{1 / 2}^{+}=\sum_{j} \mathfrak{p}^{+j / 2} \\
\mathfrak{p}^{+}=\mathfrak{p}_{1}^{+}+\mathfrak{p}_{1 / 2}^{+},
\end{gathered}
$$


where $\mathfrak{n}^{+j k}, \mathfrak{n}^{+j}$ are root spaces in $\mathfrak{g}$ for $\frac{1}{2}\left(\gamma_{j}+\gamma_{k}\right)$ and $\gamma_{j}$, respectively, and $\mathfrak{p}^{+j / 2}$ is the root space in $\mathfrak{p}^{+}\left(\subset \mathfrak{g}^{\mathbb{C}}\right)$ for $\frac{1}{2} \gamma_{j}$. Furthermore,

$$
\begin{gathered}
\mathfrak{n}_{K}=\sum_{j<k} \mathfrak{n}_{K}^{j k}, \quad \overline{\mathfrak{n}}_{K}=\sum_{j>k} \mathfrak{n}_{K}^{j k}, \\
\mathfrak{k}_{T}^{*}=i \mathfrak{h}^{-}+\mathfrak{m}+\mathfrak{n}_{K}+\overline{\mathfrak{n}}_{K}, \\
\mathfrak{k}^{\mathbb{C}}=\left(\mathfrak{k}_{T}^{*}\right)^{\mathbb{C}}+\sum_{j} \mathfrak{k}^{j / 2}+\sum_{j} \mathfrak{k}^{-j / 2},
\end{gathered}
$$

where $\mathfrak{n}_{K}^{j k}$ is the root space for $\frac{1}{2}\left(\gamma_{j}-\gamma_{k}\right)$ in $\mathfrak{g}$ and $\mathfrak{k}^{ \pm j / 2}$ is the root space for $\pm \frac{1}{2} \gamma_{j}$ in $\mathfrak{k}^{\mathbb{C}}$. (The spaces $\mathfrak{p}^{ \pm j / 2}, \mathfrak{k}^{ \pm j / 2}$ do not meet $\mathfrak{g}$; the $\frac{1}{2} \gamma_{j}$-root space in $\mathfrak{g}$ is a real form of $\mathfrak{p}^{+j / 2}+\mathfrak{k}^{+j / 2}$.)

3.4. Koecher norm function. Let $n=\operatorname{dim}_{\mathbb{C}} \mathfrak{p}^{+}, n_{1}=\operatorname{dim}_{\mathbb{C}} \mathfrak{p}_{1}^{+}$. Now $r$ is the rank of $\Omega$, and $n_{1}$ is the (real) dimension of $J$. A dimension count gives

$$
\begin{gathered}
n_{1}=\frac{r(r-1)}{2} \nu+r, \\
n=n_{1}+r b .
\end{gathered}
$$

Set

$$
\mu=n_{1} / r .
$$

The number $\mu$ is an integer or half-integer, and

$$
\mu=\frac{r-1}{2} \nu+1
$$

With respect to the inner product $(\cdot \mid \cdot)$, the characteristic function $\phi$ of $\Omega$ is defined by

$$
\phi(x)=\int_{\Omega} e^{-(x \mid y)} d y
$$

for $x \in \Omega$, from which the Koecher norm function $\Delta$ on $\Omega$ is given by

$$
\Delta(x)=c \phi(x)^{-1 / \mu}
$$

where $c$ is a constant chosen so that $\Delta(e)=1$.

\subsection{The polar decomposition of $J$. Let}

$$
\Re=\left\{s=\sum_{j=1}^{r} s_{j} e_{j}: s_{j} \in \mathbb{R}\right\}
$$

and

$$
\Re^{+}=\left\{s \in \Re: s_{1}>\cdots>s_{r}\right\} .
$$

Any element $x \in J=\mathfrak{n}_{1}^{+}$can be written as

$$
x=l \cdot s
$$


where $s \in \bar{\Re}^{+}$is unique and $l \in L_{T}^{0}$ is unique modulo the centralizer of $\Re$ in $L_{T}^{0}$. Then

$$
\Delta\left(l \cdot \sum_{j=1}^{r} s_{j} e_{j}\right)=\prod_{j=1}^{r} s_{j} .
$$

The mapping $L_{T}^{0} \times \bar{\Re}^{+} \rightarrow J$ given by (3) defines the polar decomposition for $J$. The corresponding integral formula is

$$
\int_{J} f(x) d x=c \int_{\Re^{+}}\left(\int_{L_{T}^{0}} f(l \cdot s) d l\right) \prod_{j<k}\left(s_{j}-s_{k}\right)^{\nu} d s_{1} \cdots d s_{r}
$$

where $d x$ is the Euclidean volume element on $J, d l$ is the normalized Haar measure on $L_{T}^{0}, c$ is a positive constant depending only upon $J$, and $f$ is any integrable function.

Similarly, let $d x$ again be the Euclidean volume element on $\Omega$ and $d l$ the normalized Haar measure on $L_{T}^{0}$, then

$$
\int_{\Omega} f(x) d x=c_{0} \int_{0}^{\infty} \cdots \int_{0}^{\infty} \int_{L_{T}^{0}} f\left(l \cdot \sum_{1}^{r} t_{j} e_{j}\right) d l \prod_{j<k}\left|t_{j}-t_{k}\right|^{\nu} d t_{1} \cdots d t_{r}
$$

where $c_{0}$ is also a constant.

Let $d z$ be the Euclidean volume element on $\mathfrak{p}^{+}$and $d k$ the normalized Haar measure on $K$, then

$$
\begin{aligned}
\int_{\mathfrak{p}^{+}} f(z) d z=c & \int_{0}^{\infty} \cdots \int_{0}^{\infty} \int_{K} f\left(k \cdot \sum_{1}^{r} t_{j} e_{j}\right) d k 2^{r} \\
& \times \prod_{j} t_{j}^{2 b+1} \prod_{j<k}\left|t_{j}^{2}-t_{k}^{2}\right|^{\nu} d t_{1} \cdots d t_{r}
\end{aligned}
$$

where $c$ is a constant.

3.6. Generating elements of $G$. As in [Do1], we decompose $\mathfrak{g}$ as a canonical graduation

$$
\mathfrak{g}=\mathfrak{g}_{-1} \oplus \mathfrak{g}_{-\frac{1}{2}} \oplus \mathfrak{g}_{0} \oplus \mathfrak{g}_{\frac{1}{2}} \oplus \mathfrak{g}_{1}
$$

Then $\mathfrak{k}_{T}^{*}$ in Section 3.2 coincides with $\mathfrak{g}_{0}(\mathrm{cf} .[\mathrm{M}]), \mathfrak{n}_{1}^{ \pm}$coincides with $\mathfrak{g}_{ \pm 1}$,

$$
\mathfrak{g}_{-\frac{1}{2}}=\sum_{i=1}^{r} \mathfrak{g}_{-\frac{1}{2} \gamma_{i}},
$$

and

$$
\mathfrak{g}_{\frac{1}{2}}=\sum_{i=1}^{r} \mathfrak{g}_{\frac{1}{2} \gamma_{i}},
$$

where $\mathfrak{g}_{ \pm \frac{1}{2} \gamma_{i}}$ are the root spaces in $\mathfrak{g}$ for $\pm \frac{1}{2} \gamma_{i}$.

We adopt a notation $(z, u)$ for the element of $V_{2} \oplus V_{1}$, where $z \in V_{2}$ and $u \in V_{1}$; i.e., $(z, u)=z+u$. Denote $G_{-1}, G_{-\frac{1}{2}}, G_{0}, G_{\frac{1}{2}}$, and $G_{1}$ as the corresponding analytic subgroups. Viewing $G$ as an automorphism group of the domain $\Sigma$ given by (2.7.1), $G_{0}, G_{\frac{1}{2}}$, and $G_{1}$ act on $\Sigma$ as follows:

(a) Elements $a$ in $G_{0}$ can be described as

$$
a \cdot(z, u)=(a z, a u) ;
$$


i.e., as an operator on $V_{2}, a \in G L\left(V_{2}\right)$ and $a \Omega=\Omega$; as an operator on $V_{1}, a \in$ $G L\left(V_{1}\right)$; moreover,

$$
a B(u, \bar{u})=B(a u, \overline{a u}) .
$$

(b) Elements in $G_{\frac{1}{2}}$ can be parametrized by elements $u_{0}$ in $V_{1}$; i.e., for $u_{0} \in V_{1}$

$$
R\left(u_{0}\right) \cdot(z, u)=\left(z+i B\left(u, \bar{u}_{0}\right)+\frac{i}{2} B\left(u_{0}, \bar{u}_{0}\right), u+u_{0}\right) .
$$

(c) Elements in $G_{1}$ can be parametrized by elements $x_{0}$ in $J$; i.e., for $x_{0} \in J$

$$
R\left(x_{0}\right) \cdot(z, u)=\left(z+x_{0}, u\right)
$$

By [Do1, Corollary 3.6] the symmetry $g_{i e}$ of the Siegel domain $\Sigma$ with respect to the point $i e$ is given by

$$
g_{i e} \cdot(z, u)=\left(-z^{-1},-i z^{-1} \circ u\right),
$$

where $z \circ u$ is given by (2.7.2). To simplify the discussion, we study the element $j$ given by

$$
j \cdot(z, u)=\left(-z^{-1},-z^{-1} \circ u\right) .
$$

Denote by $\theta$ the Cartan involution with respect to the point $i e$, then $\theta=A d\left(g_{i e}\right)$. By [Do1], $\theta \mathfrak{g}_{\lambda}=\mathfrak{g}_{-\lambda}$ for $\lambda \in\left\{-1,-\frac{1}{2}, 0, \frac{1}{2}, 1\right\}$. By (1), elements $a, R\left(u_{0}\right), R\left(x_{0}\right)$ and $g_{i e}$ generate the group $G$. Then by (8), $a, R\left(u_{0}\right), R\left(x_{0}\right)$ and $j$ generate the group $G$.

3.7. The multiplier $m$. For $g \in G$ denote by $\frac{\partial g \cdot(z, u)}{\partial(z, u)}$ the Jacobi at $(z, u) \in \Sigma$; i.e.,

$$
\frac{\partial g \cdot(z, u)}{\partial(z, u)}(w, v)=\lim _{h \rightarrow 0} \frac{g \cdot(z+h w, u+h v)-g \cdot(z, u)}{h}
$$

for $(w, v) \in V_{2} \oplus V_{1}$, where $h$ is a complex scalar. Define

$$
m(g,(z, u))=\left(\frac{\partial g^{-1} \cdot(z, u)}{\partial(z, u)}\right)^{-1}
$$

for $(g,(z, u)) \in G \times \Sigma$. Then $m$ satisfies the multiplier identity

$$
m\left(g_{1} g_{2},(z, u)\right)=m\left(g_{1},(z, u)\right) m\left(g_{2}, g_{1}^{-1} \cdot(z, u)\right)
$$

for $g_{1}, g_{2} \in G$ and $(z, u) \in \Sigma$. The definitions (1) and (2) can be generalized to $g \in G^{\mathbb{C}},(z, u) \in V_{2} \oplus V_{1}$, and the property (3) is still valid.

We compute $m$ on the set of generators $a, R\left(u_{0}\right), R\left(x_{0}\right), j$ of $G$ given by (3.6.4), (3.6.6), (3.6.7) and (3.6.8).

3.8. Proposition. For the generators of $G$ given by (3.6.4), (3.6.6), (3.6.7) and (3.6.8),

$$
\begin{gathered}
m(a,(z, u))=a, \\
m\left(R\left(u_{0}\right),(z, u)\right) \cdot(w, v)=\left(w+i B\left(v, \bar{u}_{0}\right), v\right), \\
m\left(R\left(x_{0}\right),(z, u)\right)=I, \\
m(j,(z, u)) \cdot(w, v)=(P(z) w, z \circ v+z \circ(w \circ u)) .
\end{gathered}
$$


Proof. (1) and (3) follow from direct calculations. Since

$$
\begin{aligned}
& \frac{\partial\left(R\left(u_{0}\right) \cdot(z, u)\right)}{\partial(z, u)}(w, v) \\
& =\lim _{h \rightarrow 0}\left(\frac{z+h w+i B\left(u+h v, \bar{u}_{0}\right)+\frac{i}{2} B\left(u_{0}, \bar{u}_{0}\right)-z-i B\left(u, \bar{u}_{0}\right)-\frac{i}{2} B\left(u_{0}, \bar{u}_{0}\right)}{h},\right. \\
& =\left(w+i B\left(v, \bar{u}_{0}\right), v\right),
\end{aligned}
$$

we have (2). By [Koe, p. 64],

$$
\begin{aligned}
& \frac{\partial\left(j^{-1} \cdot(z, u)\right)}{\partial(z, u)}(w, v) \\
& =\lim _{h \rightarrow 0}\left(\frac{-(z+h w)^{-1}+z^{-1}}{h}, \frac{(z+h w)^{-1} \circ(u+h v)-z^{-1} \circ u}{h}\right) \\
& =\left(P(z)^{-1} w, z^{-1} \circ v-\left(P(z)^{-1} w\right) \circ u\right) .
\end{aligned}
$$

Then (4) follows from (3.7.2).

For $z_{0} \in V_{2}$, define a mapping $r\left(z_{0}\right)$ by

$$
r\left(z_{0}\right):(z, u) \mapsto\left(P\left(z_{0}\right) z, z_{0} \circ u\right) .
$$

3.9. Proposition. Operators a, $m\left(R\left(u_{0}\right),(z, u)\right), m\left(R\left(x_{0}\right),(z, u)\right), m(j,(z, u))$ given by (3.8.1)-(3.8.4) are elements in $K^{\mathbb{C}}$.

Proof. By Section 3.6, $a \in K_{T}^{*} \subset K_{T}^{\mathbb{C}} \subset K^{\mathbb{C}}$. Denote

$$
\mathfrak{k}_{\frac{1}{2}}=\sum_{j=1}^{r} \mathfrak{k}^{j / 2}, \quad \mathfrak{k}_{-\frac{1}{2}}=\sum_{j=1}^{r} \mathfrak{k}^{-j / 2},
$$

and $K_{\frac{1}{2}}, K_{-\frac{1}{2}}$ as the corresponding subgroups. Then $K_{-\frac{1}{2}}$ can be parametrized by elements $u_{0} \in V_{1}$; i.e.,

$$
k\left(u_{0}\right):(z, u) \mapsto\left(z, z \circ u_{0}+u\right)
$$

where $u_{0} \in V_{1}$. By $[\mathrm{L} 1,3.4,3.5], k\left(u_{0}\right)^{*}$ is the map

$$
k\left(u_{0}\right)^{*}:(z, u) \mapsto\left(z+B\left(u, \bar{u}_{0}\right), u\right),
$$

and $k\left(u_{0}\right)^{*} \in K_{\frac{1}{2}}$. Thus, $(1)$ and $(2)$ are in $K^{\mathbb{C}}$, so is $m\left(R\left(u_{0}\right),(z, u)\right)$. It is obvious that $m\left(R\left(x_{0}\right),(z, u)\right) \in K^{\mathbb{C}}$.

By [L1], 10.11(2), $r\left(x_{0}\right)$ given by (3.8.5) is in $K_{T}^{*}$ for invertible $x \in J$. Consequently, $r\left(z_{0}\right) \in K^{\mathbb{C}}$ for all invertible $z_{0} \in V_{2}$. If $(z, u) \in \Sigma$, then $\operatorname{Im} z>0$ and $z$ is invertible. By (1) and (3.8.4), $m(j,(z, u)) \in K^{\mathbb{C}}$ and the proposition is proved.

3.10. Decompositions of $A$. We now study the connected components of the identity in $K_{T}^{*}$ and $L_{T}$, and denote them by $A$ and $L$, respectively. Thus, $L_{T}^{0}=L$. We also denote $N=N_{K}$. Then the group $A$ has a polar decomposition; i.e., each $a \in A$ can be written uniquely as

$$
a=r\left(\xi^{1 / 2}\right) l
$$


where $\xi \in \Omega, \xi^{1 / 2}$ is its unique root in $\Omega, l \in L$, and $r(\cdot)$ is given by (3.8.5). Relative to this polar decomposition, the Haar measure on $A$ decomposes as

$$
d a=d_{*} \xi d l
$$

where $d_{*} \xi=\Delta(\xi)^{-\mu} d \xi$ is an $A$-invariant measure on the cone $\Omega$ and $d l$ is the normalized Haar measure on $L$.

Related to the polar decomposition (1) is the Iwasawa decomposition $A=H N L$, where $H=\exp \left(i \mathfrak{h}^{-1}\right)$ and $N$ are the analytic subgroups having Lie algebras $i \mathfrak{h}^{-1}$ and $\mathfrak{n}_{K}$, respectively, given in Section 3.3. Each $a \in A$ has a unique decomposition $a=h n l$ with $(h, n, l)$ in $H \times N \times L$. The group $N$ is nilpotent, $H$ is abelian, and $H$ normalizes $N$. The decomposition of Haar measure on $A$ with respect to this decomposition is

$$
d a=d h d n d l
$$

where $d h, d n$ and $d l$ denote Haar measures on $H, N$, and $L$, respectively.

Relative to a choice of basis, the root spaces $\mathfrak{n}_{K}^{j k}$ consist of vectors $q_{j k} \in \mathbb{R}^{\nu}$. Since the exponential map is a bijection of $\mathfrak{n}_{K}$ with $N$, the coordinates $q_{j k} \in \mathbb{R}^{\nu}$ for $1 \leq j<k \leq r$ parametrize $N$. Similarly, the mapping $\left(q_{1}, \cdots q_{r}\right) \mapsto h=$ $\exp \left(\sum_{j=1}^{r}\left(\log q_{j}\right) h_{j}\right)$ is an isomorphism of the multiplicative group $\left(\mathbb{R}^{+}\right)^{r}$ with $H$. In these coordinates,

$$
d h=\prod_{i=1}^{r} \frac{d q_{i}}{q_{i}} \quad \text { and } \quad d n=d n(q)=\prod_{j=1}^{r} \prod_{k=1}^{j-1} d q_{j k},
$$

respectively. The group $B=H N$ acts simply transitively on $\Omega$, which implies that the mapping $b \mapsto x=b \cdot e$ is a diffeomorphism of $B$ with $\Omega$. Then

$$
\Delta(x)=\Delta(h \cdot e)=\prod_{j=1}^{r} q_{j}^{2} \quad \text { and } \quad(x \mid e)=(b \cdot e \mid e)=\sum_{j=1}^{r} q_{j}^{2}\left(1+\sum_{k=1}^{j-1}\left\|q_{j k}\right\|^{2}\right)
$$

where $\|\cdot\|$ denotes the usual Euclidean norm in $\mathbb{R}^{\nu}$.

\section{Weighted Laplace transforms}

In this section, we define the operator-valued gamma functions for a Siegel domain $\Sigma$ of type II by an integral analogous to the integral formula for the classical gamma function, and index them by irreducible representations $\pi$ of the group $K^{\mathbb{C}}$. We study a weighted Laplace transform on $\Omega \oplus V_{1}$, its inversion formula, and the Plancherel formula for this transform.

4.1. The restricted lowest weight. Let $\pi$ be an irreducible finite-dimensional holomorphic (ifdh) representation of $K^{\mathbb{C}}, \mathcal{V}=\mathcal{V}_{\pi}$ be the space on which $\pi$ acts, and denote by $\mathcal{E}=\mathcal{E}_{\pi}$ the space of linear transformations on $\mathcal{V}_{\pi}$. Endow $\mathcal{E}$ with the inner product

$$
(S \mid T)=\operatorname{tr}\left(T^{*} S\right) .
$$

The representation $\pi$ is characterized by its lowest weight, and the restriction of the lowest weight to $i \mathfrak{h}^{-1}$ is determined by its signature. The signature is an $r$-tuple $M=\left(m_{1}, m_{2}, \cdots m_{r}\right)$ of integers such that

$$
m_{1} \geq m_{2} \geq \cdots \geq m_{r} .
$$


At the Lie algebra level, the signature corresponds to the linear functional

$$
\Lambda_{M}=-\frac{1}{2} \sum_{j=1}^{r} m_{j} \gamma_{j}
$$

which is called the restricted lowest weight of $\pi$.

The lowest weight becomes a character $\chi_{M}$ of $H$, called the lowest weight character of $\pi$ on $H$. If we use the coordinates and write $h=\exp \left(\sum_{j=1}^{r}\left(\log q_{j}\right) h_{j}\right)$, then

$$
\chi_{M}(h)=q_{1}^{-m_{1}} q_{2}^{-m_{2}} \cdots q_{r}^{-m_{r}} .
$$

We designate the last (and smallest) entry in the signature by the notation

$$
\omega(\pi)=m_{r} .
$$

4.2. Notation. By the proof of Proposition 3.9, if $z \in V_{2}$ is invertible, then $r(z) \in K_{T}^{\mathbb{C}}$. Thus, $\pi(r(z))$ is defined for all invertible $z \in V_{2}$. For convenience we adopt the notation $\pi(z)$ rather than the more cumbersome $\pi(r(z))$; i.e., by definition,

$$
\pi(z)=\pi(r(z))
$$

for all invertible elements $z \in V_{2}$. In particular, $\pi(\xi)$ is defined for all $\xi \in \Omega$ and $\pi(z)$ is defined for all $z \in V_{2}$ with $\operatorname{Im} z>0$.

Note that $A$ normalizes $K_{-\frac{1}{2}}$, and $A K_{-\frac{1}{2}}$ is a subgroup of $K^{\mathbb{C}}$.

4.3. Definition. Let $\Delta$ be defined by (3.4.6). For $\alpha \in \mathbb{C}$ and $(z, u) \in \Sigma$, set

$$
\begin{aligned}
\Gamma((z, u), \alpha, \pi) & =\int_{A K_{-\frac{1}{2}}} e^{2 i(a z \mid e)} e^{2\left(a u \mid u_{0}\right)} e^{-\left(u_{0} \mid u_{0}\right)} \\
& \times \pi\left(\left(a k\left(i u_{0}\right)\right)^{*} a k\left(i u_{0}\right)\right)^{-1} \Delta(a e)^{\alpha} d a d u_{0} .
\end{aligned}
$$

Whenever the integral (1) converges absolutely, the mapping $((z, u), \alpha, \pi) \mapsto$ $\Gamma((z, u), \alpha, \pi)$ is called the (generalized) gamma function for the domain $\Sigma$.

In general, the gamma function is operator-valued; that is, $\Gamma((z, u), \alpha, \pi) \in \mathcal{E}_{\pi}$. When $(z, u)=\left(\frac{i e}{2}, 0\right)$, we set

$$
\begin{aligned}
& \Gamma_{\pi}(\alpha)=\Gamma\left(\left(\frac{i e}{2}, 0\right), \alpha, \pi\right) \\
& =\int_{A K_{-\frac{1}{2}}} e^{-(a e \mid e)} e^{-\left(u_{0} \mid u_{0}\right)} \pi\left(\left(a k\left(i u_{0}\right)\right)^{*} a k\left(i u_{0}\right)\right)^{-1} \Delta(a e)^{\alpha} d a d u_{0}
\end{aligned}
$$

and refer to the $\mathcal{E}_{\pi}$-valued mapping $\alpha \mapsto \Gamma_{\pi}(\alpha)$ as the gamma function for $\Omega$ of weight $\pi$.

By (3.10.1), we write $a=r\left(\xi^{\frac{1}{2}}\right) l$, and then $a a^{*}=r(\xi)$. By (3.10.2) we can write (2) as

$$
\Gamma_{\pi}(\alpha)=\int_{\Omega \oplus V_{1}} e^{-(\xi \mid e)} e^{-\left(u_{0} \mid u_{0}\right)} \pi\left(k\left(i u_{0}\right)^{*} r(\xi) k\left(i u_{0}\right)\right)^{-1} \Delta(\xi)^{\alpha-\mu} d \xi d u_{0} .
$$

4.4. Theorem. Let $\alpha \in \mathbb{C}$ and $(z, u) \in \Sigma$. Then the integral defining $\Gamma((z, u), \alpha, \pi)$ converges absolutely if and only if

$$
\omega(\pi)>\mu-\operatorname{Re} \alpha-1,
$$

where $\mu$ is given by (3.4.3). 
Proof. Without loss of generality, we assume $\alpha$ is real. By (4.1.1)

$$
\operatorname{tr}\left(\pi\left(\left(a k\left(i u_{0}\right)\right)^{*} a k\left(i u_{0}\right)\right)\right)=\left\|\pi\left(a k\left(i u_{0}\right)\right)\right\|^{2} .
$$

By consideration of eigenvalues

$$
\begin{aligned}
\left\|\pi\left(\left(a k\left(i u_{0}\right)\right)^{*} a k\left(i u_{0}\right)\right)\right\| & \leq \operatorname{tr}\left(\pi\left(\left(a k\left(i u_{0}\right)\right)^{*} a k\left(i u_{0}\right)\right)\right) \\
\leq & d^{1 / 2}\left\|\pi\left(\left(a k\left(i u_{0}\right)\right)^{*} a k\left(i u_{0}\right)\right)\right\|,
\end{aligned}
$$

where $d$ is the dimension of $\mathcal{V}_{\pi}$. Hence, it suffices to consider the convergence of

$$
\int_{A K_{-\frac{1}{2}}}\left|e^{2 i(a z \mid e)} e^{2\left(a u \mid u_{0}\right)} e^{-\left(u_{0} \mid u_{0}\right)}\right|\left\|\pi\left(a k\left(i u_{0}\right)\right)^{-1}\right\|^{2} \Delta(a e)^{\alpha} d a d u_{0} .
$$

Since $(z, u) \in \Sigma, \quad t=\operatorname{Im} z-\frac{1}{2} B(u, \bar{u})>0$ (cf. (2.7.1)). By [L1], Sections 3.4 and 3.5 ,

$$
(u \mid v)=(B(u, \bar{v}) \mid e)
$$

for $u, v \in V_{1}$. By (3) and (3.6.5),

$$
(a u \mid a u)=(B(a u, \overline{a u}) \mid e)=(a B(u, \bar{u}) \mid e) .
$$

Then (2) is equal to

$$
\begin{aligned}
& \int_{A K_{-\frac{1}{2}}} e^{-2(a t \mid e)} e^{-(a B(u, \bar{u}) \mid e)}\left|e^{2\left(a u \mid u_{0}\right)}\right| e^{-\left(u_{0} \mid u_{0}\right)} \| \pi\left(a k\left(i u_{0}\right)\right)^{-1}||^{2} \Delta(a e)^{\alpha} d a d u_{0} \\
& =\int_{A K_{-\frac{1}{2}}} e^{-2\left(t \mid a^{*} e\right)} e^{-\left(u_{0}-a u \mid u_{0}-a u\right)}\left\|\pi\left(a k\left(i u_{0}\right)\right)^{-1}\right\|^{2} \Delta(a e)^{\alpha} d a d u_{0}
\end{aligned}
$$

or

$$
\int_{K_{-\frac{1}{2}}}\left[\int_{A} e^{-2\left(t \mid a^{*} e\right)}\left\|\pi\left(a k\left(i\left(u_{0}+a u\right)\right)\right)^{-1}\right\|^{2} \Delta(a e)^{\alpha} d a\right] e^{-\left(u_{0} \mid u_{0}\right)} d u_{0}
$$

We first assume that (5) is convergent, then so is the integral

$$
\int_{K_{-\frac{1}{2}}}\left[\int_{A} e^{-2\left(t \mid a^{*} e\right)} e^{-2(a u \mid a u)}\left\|\pi\left(a k\left(i u_{0}\right)\right)^{-1} f\right\|^{2} \Delta(a e)^{\alpha} d a\right] e^{-2\left(u_{0} \mid u_{0}\right)} d u_{0}
$$

for $f \in \mathcal{V}_{\pi}$, since $\left(u_{0}-a u \mid u_{0}-a u\right) \leq 2\left(u_{0} \mid u_{0}\right)+2(a u \mid a u)$. Let $f$ be a unit lowest weight vector of $\pi$ corresponding to the lowest weight $-\frac{1}{2} \sum_{j=1}^{r} m_{j} \gamma_{j}$, then

$$
\left\|\pi\left(a k\left(i u_{0}\right)\right)^{-1} f\right\|=\left\|\pi(a)^{-1} f\right\| .
$$

By (7) and the Fubini theorem,

$$
\int_{A} e^{-2\left(t \mid a^{*} e\right)} e^{-2(a u \mid a u)}\left\|\pi(a)^{-1} f\right\|^{2} \Delta(a e)^{\alpha} d a
$$

is convergent. 
By (3.10.4), (3.10.5) and change of variables $q_{j k} \mapsto q_{j}^{-1} q_{j k}$, we obtain

$$
\begin{aligned}
\int_{N} e^{-(h n e \mid e)} d n & =\prod_{j=1}^{r} \prod_{k=1}^{j-1}\left(\int_{\mathbb{R}^{\nu}} e^{-q_{j}^{2}\left(1+\left\|q_{j k}\right\|^{2}\right)} d q_{j k}\right) \\
& =\prod_{j=1}^{r}\left(e^{-q_{j}^{2}} \int_{\mathbb{R}^{\nu}} \prod_{k=1}^{j-1} e^{-\left\|q_{j} q_{j k}\right\|^{2}} d q_{j k}\right) \\
& =c \eta(q) e^{-\|q\|^{2}}
\end{aligned}
$$

where $h=h(q)=h\left(q_{1}, \cdots, q_{r}\right), c$ is the constant

$$
c=\pi^{r(r-1) \nu / 4}=\prod_{k<j} \int_{\mathbb{R}^{\nu}} e^{-\left\|q_{j k}\right\|^{2}} d q_{j k}
$$

and $\eta$ is the Jacobian

$$
\eta(q)=\left(q_{2} q_{3}^{2} \cdots q_{r}^{r-1}\right)^{-\nu} .
$$

By (4), the integral (8) is equal to

$$
\int_{A} e^{-2(a(t+B(u, \bar{u})) \mid e)}\left\|\pi(a)^{-1} f\right\|^{2} \Delta(a e)^{\alpha} d a .
$$

Since it is convergent, so is the integral

$$
\int_{A} e^{-(a e \mid e)}\left\|\pi(a)^{-1} f\right\|^{2} \Delta(a e)^{\alpha} d a .
$$

By (3.10.3), (3.10.4), (3.10.5), (4.1.4), (9), (10) and (11), it is equal to

$$
\begin{aligned}
& c \int_{H} e^{-\|q\|^{2}} \eta(q) \chi_{M}(h(q))^{-2} \Delta(h(q) e)^{\alpha} d h(q) \\
& =c \prod_{j=1}^{r} \int_{0}^{\infty} e^{-q_{j}^{2}} q_{j}^{2\left(m_{j}+\alpha\right)-\nu(j-1)-1} d q_{j} \\
& =c 2^{-r} \prod_{j=1}^{r} \Gamma\left(m_{j}+\alpha-(j-1) \nu / 2\right) .
\end{aligned}
$$

Since it is convergent, $m_{r}+\alpha-(r-1) \nu / 2>0$ and (1) follows from (3.4.4).

Conversely, if (1) is valid, then the restriction of $\pi$ to $A$ is generally reducible. By Cartan's Theorem for the lowest weight [Kn], Proposition 4.34, any irreducible component $\pi^{\prime}$ of this restriction has the lowest weight

$$
\Lambda_{b}=-\frac{1}{2} \sum_{j=1}^{r} b_{j} \gamma_{j}
$$

and $b_{r} \geq m_{r}=\omega(\pi)$. Then

$$
b_{r}>\mu-\operatorname{Re} \alpha-1,
$$

and by the same calculation as one in the last paragraph (cf. [DG1], Theorem 2.5)

$$
\int_{A} e^{-2\left(t \mid a^{*} e\right)}\left\|\pi^{\prime}(a)^{-1}\right\|^{2} \Delta(a e)^{\alpha} d a<\infty,
$$


and hence

$$
\int_{A} e^{-2\left(t \mid a^{*} e\right)}\left\|\pi(a)^{-1}\right\|^{2} \Delta(a e)^{\alpha} d a<\infty .
$$

By [RV1, Proposition 5.6(b)], $\pi(k(i u))$ is a polynomial in $u$. Then the integral

$$
\int_{A} e^{-2\left(t \mid a^{*} e\right)}\left\|\pi\left(a k\left(i\left(u_{0}+a u\right)\right)\right)^{-1}\right\|^{2} \Delta(a e)^{\alpha} d a,
$$

and the integral (6) are also convergent. The theorem is now proved.

4.5. Corollary. Let $\pi$ be an ifdh representation of $K^{\mathbb{C}}$ and $\alpha$ be a real number such that

$$
\omega(\pi)>\mu-\alpha-1 .
$$

Then $\Gamma_{\pi}(\alpha)$ is a positive definite linear transformation on $\mathcal{V}_{\pi}$. Moreover,

$$
\pi(l) \Gamma_{\pi}(\alpha)=\Gamma_{\pi}(\alpha) \pi(l)
$$

for all $l \in L$.

4.6. Weighted Laplace transforms. As in Section 4.1, let $\pi$ be an ifdh representation of $K^{\mathbb{C}}$, let $\mathcal{V}_{\pi}$ be the space of $\pi$, and let $\mathcal{E}$ be the space of linear transformations on $\mathcal{V}_{\pi}$. Let $\phi: \Omega \oplus V_{1} \rightarrow \mathcal{E}$ be a measurable function such that it is holomorphic in $V_{1}$ and the integral

$$
\Phi(z, u)=\pi^{-\frac{n}{2}} \int_{\Omega \oplus V_{1}} e^{i(z \mid \xi)} e^{\left(\xi^{\frac{1}{2}} \circ u \mid u_{0}\right)} e^{-\left(u_{0} \mid u_{0}\right)} \pi\left(r\left(\xi^{\frac{1}{2}}\right) k\left(i u_{0}\right)\right)^{*^{-1}} \phi\left(\xi, u_{0}\right) d \xi d u_{0}
$$

converges absolutely for all $(z, u) \in \Sigma$. We call the function $\Phi$ the $\pi$-weighted Laplace transform of $\phi$.

By the definition (2.7.1) of $\Sigma$, any element in $\Sigma$ can be expressed by $(x+i y, u)$, where $y=t+\frac{1}{2} B(u, \bar{u})$ with $t>0$.

4.7. Proposition. Whenever the function $x \mapsto \Phi(x+i y, u)$ is integrable,

$$
\begin{aligned}
\phi\left(\xi, u_{0}\right) & =2^{-n_{1}} \pi^{-\left(\frac{n}{2}+r b\right)} \int_{J \oplus V_{1}} e^{-i\left(x+i\left(t+\frac{1}{2} B(u, \bar{u})\right) \mid \xi\right)} e^{\left(u_{0} \mid \xi^{1 / 2} \circ u\right)} e^{-\left(\xi^{1 / 2} \circ u \mid \xi^{1 / 2} \circ u\right)} \\
& \times \Delta(\xi)^{b} \pi\left(r\left(\xi^{\frac{1}{2}}\right) k\left(i \xi^{1 / 2} \circ u\right)\right)^{*} \Phi\left(x+i\left(t+\frac{1}{2} B(u, \bar{u})\right), u\right) d x d u,
\end{aligned}
$$

where the integral is independent of the choice of $t>0$. The formula (1) is called the inverse formula of the $\pi$-weighted Laplace transform (4.6.1).

Proof. By (4.6.1)

$$
\begin{aligned}
& 2^{-n_{1}} \pi^{-\left(\frac{n}{2}+r b\right)} \int_{J \oplus V_{1}} e^{-i\left(x+i\left(t+\frac{1}{2} B(u, \bar{u})\right) \mid \xi\right)} e^{\left(u_{0} \mid \xi^{\frac{1}{2}} \circ u\right)} e^{-\left(\xi^{\frac{1}{2}} \circ u \mid \xi^{\frac{1}{2}} \circ u\right)} \\
& \times \Delta(\xi)^{b} \pi\left(r\left(\xi^{\frac{1}{2}}\right) k\left(i \xi^{\frac{1}{2}} \circ u\right)\right)^{*} \Phi\left(x+i\left(t+\frac{1}{2} B(u, \bar{u})\right), u\right) d x d u \\
& =2^{-n_{1}} \pi^{-(n+r b)} \int_{J \oplus V_{1}} e^{-i\left(x+i\left(t+\frac{1}{2} B(u, \bar{u})\right) \mid \xi\right)} e^{\left(u_{0} \mid \xi^{\frac{1}{2}} \circ u\right)} e^{-\left(\xi^{\frac{1}{2}} \circ u \mid \xi^{\frac{1}{2}} \circ u\right)} \\
& \times \Delta(\xi)^{b} \pi\left(r\left(\xi^{\frac{1}{2}}\right) k\left(i \xi^{\frac{1}{2}} \circ u\right)\right)^{*}\left[\int_{\Omega \oplus V_{1}} e^{i\left(x+i\left(t+\frac{1}{2} B(u, \bar{u})\right) \mid \xi^{\prime}\right)}\right. \\
& \left.\times e^{\left(\xi^{\prime \frac{1}{2}} \circ u \mid u_{0}^{\prime}\right)} e^{-\left(u_{0}^{\prime} \mid u_{0}^{\prime}\right)} \pi\left(r\left(\xi^{\prime \frac{1}{2}}\right) k\left(i u_{0}^{\prime}\right)\right)^{*-1} \phi\left(\xi^{\prime}, u_{0}^{\prime}\right) d \xi^{\prime} d u_{0}^{\prime}\right] d x d u .
\end{aligned}
$$


By the classical inverse Laplace transform formula (cf. [RV1]),

$$
\begin{gathered}
(2 \pi)^{-n_{1}} \int_{J} e^{-i\left(x+i\left(t+\frac{1}{2} B(u, \bar{u})\right) \mid \xi\right)}\left[\int_{\Omega} e^{i\left(x+i\left(t+\frac{1}{2} B(u, \bar{u})\right) \mid \xi^{\prime}\right)}\right. \\
\left.\times e^{\left(\xi^{\prime \frac{1}{2}} \circ u \mid u_{0}^{\prime}\right)} \pi\left(r\left(\xi^{\prime \frac{1}{2}}\right) k\left(i u_{0}^{\prime}\right)\right)^{*-1} \phi\left(\xi^{\prime}, u_{0}^{\prime}\right) d \xi^{\prime}\right] d x \\
=e^{\left(\xi^{\frac{1}{2}} \circ u \mid u_{0}^{\prime}\right)} \pi\left(r\left(\xi^{\frac{1}{2}}\right) k\left(i u_{0}^{\prime}\right)\right)^{*-1} \phi\left(\xi, u_{0}^{\prime}\right) .
\end{gathered}
$$

Since $n=n_{1}+r b,(2)$ is equal to

$$
\begin{aligned}
& \pi^{-2 r b} \int_{V_{1}} e^{\left(u_{0} \mid \xi^{\frac{1}{2}} \circ u\right)} e^{-\left(\xi^{\frac{1}{2}} \circ u \mid \xi^{\frac{1}{2}} \circ u\right)} \Delta(\xi)^{b} \pi\left(r\left(\xi^{\frac{1}{2}}\right) k\left(i \xi^{\frac{1}{2}} \circ u\right)\right)^{*} \\
& \times\left[\int_{V_{1}} e^{\left(\xi^{\frac{1}{2}} \circ u \mid u_{0}^{\prime}\right)} e^{-\left(u_{0}^{\prime} \mid u_{0}^{\prime}\right)} \pi\left(r\left(\xi^{\frac{1}{2}}\right) k\left(i u_{0}^{\prime}\right)\right)^{*-1} \phi\left(\xi, u_{0}^{\prime}\right) d u_{0}^{\prime}\right] d u .
\end{aligned}
$$

By the reproducing kernel property of a Fock space (cf. [Di2], Section 4), if $\phi$ is holomorphic in a complex vector space $V$ and integrable with respect to the measure $e^{-(u \mid u)} d u$ of $V$, then

$$
\phi\left(u_{0}^{\prime}\right)=\pi^{-d} \int_{V} e^{\left(u_{0}^{\prime} \mid u\right)} e^{-(u \mid u)} \phi(u) d u
$$

where $d$ is the dimension of $V$ over the complex field $\mathbb{C}$. Note that $\operatorname{dim}_{\mathbb{C}} V_{1}=r b$. By [Gi], (2.50), the Jacobian of the map $u \mapsto \xi^{\frac{1}{2}} \circ u$ on $V_{1}$ is $\Delta(\xi)^{b}$ for $\xi \in \Omega$. By (3.9.1), $k(u)$ is holomorphic in $u$, so is $e^{\left(u \mid u_{0}\right)} \pi\left(r\left(\xi^{\frac{1}{2}}\right) k(i u)\right)$. Letting $\phi(u)=$ $e^{\left(u \mid u_{0}\right)} \pi\left(r\left(\xi^{\frac{1}{2}}\right) k(i u)\right)$, changing variables $u \mapsto \xi^{\frac{1}{2}} \circ u$ and taking adjoints on both sides of (4), we have

$$
\begin{aligned}
& \pi^{-r b} \int_{V_{1}} e^{\left(u_{0} \mid \xi^{\frac{1}{2}} \circ u\right)} e^{-\left(\xi^{\frac{1}{2}} \circ u \mid \xi^{\frac{1}{2}} \circ u\right)} \Delta(\xi)^{b} \pi\left(r\left(\xi^{\frac{1}{2}}\right) k\left(i \xi^{\frac{1}{2}} \circ u\right)\right)^{*} e^{\left(\xi^{\frac{1}{2}} \circ u \mid u_{0}^{\prime}\right)} d u \\
& =e^{\left(u_{0} \mid u_{0}^{\prime}\right)} \pi\left(r\left(\xi^{\frac{1}{2}}\right) k\left(i u_{0}^{\prime}\right)\right)^{*} .
\end{aligned}
$$

Then by (4) again, (3) is equal to

$$
\pi^{-r b} \int_{V_{1}} e^{-\left(u_{0}^{\prime} \mid u_{0}^{\prime}\right)} e^{\left(u_{0} \mid u_{0}^{\prime}\right)} \phi\left(\xi, u_{0}^{\prime}\right) d u_{0}^{\prime}=\phi\left(\xi, u_{0}\right) .
$$

and (1) is proved.

We now prove a Plancherel-type theorem for the Laplace transform given by (4.6.1).

4.8. Theorem. Let $\pi, \mathcal{E}$ be given in Section 4.1, and $\alpha$ be a real number such that $\omega(\pi)+\alpha>\mu-1$. Suppose $\phi: \Omega \oplus V_{1} \rightarrow \mathcal{E}$ is a measurable function such that it is holomorphic in $V_{1}$ and the $\pi$-weighted Laplace transform $\Phi$ given by (4.6.1) is absolutely convergent for all $(z, u) \in \Sigma$. Then $\Phi$ is holomorphic in $\Sigma$ and

$$
\begin{aligned}
\int_{\Sigma} \| & \pi\left(r(2 y-B(u, \bar{u}))^{\frac{1}{2}} k(-i u)\right)^{*-1} \Phi(x+i y, u) \|^{2} \Delta(2 y-F(u, \bar{u}))^{-\mu+\alpha} d x d y d u \\
= & \int_{\Omega \oplus V_{1}}\left(\Gamma_{\pi}(\alpha) \phi\left(\xi, u_{0}\right) \mid \phi\left(\xi, u_{0}\right)\right) \Delta(\xi)^{-\alpha-b} e^{-\left(u_{0} \mid u_{0}\right)} d \xi d u_{0}
\end{aligned}
$$


Proof. That $\Phi$ is holomorphic in $\Sigma$ follows from the theory of the Laplace transform. By (3.9.1), (3.6.5) and a computation,

$$
k\left(i u_{0}\right) a=a k\left(i a^{*} u_{0}\right) .
$$

By (4.6.1), and the Plancherel formula for the functions on $J$, we have

$$
\begin{aligned}
& \int_{J}\left\|\pi\left(r(2 t)^{\frac{1}{2}} k(-i u)\right)^{*^{-1}} \Phi\left(x+i\left(t+\frac{1}{2} B(u, \bar{u})\right), u\right)\right\|^{2} d x \\
& =\pi^{-n} \int_{J} \| \int_{\Omega \oplus V_{1}} e^{i(x \mid \xi)} e^{-(t \mid \xi)} e^{-\frac{1}{2}(B(u, \bar{u}) \mid \xi)} e^{\left(\xi^{\frac{1}{2}} \circ u \mid u_{0}\right)} e^{-\left(u_{0} \mid u_{0}\right)} \\
& \quad \times \pi\left(r(2 t)^{\frac{1}{2}} k(-i u)\right)^{*^{-1}} \pi\left(r\left(\xi^{\frac{1}{2}}\right) k\left(i u_{0}\right)\right)^{*^{-1}} \phi\left(\xi, u_{0}\right) d \xi d u_{0} \|^{2} d x \\
& =2^{n_{1}} \pi^{-r b} \int_{\Omega} \| \int_{V_{1}} e^{-(t \mid \xi)} e^{-\frac{1}{2}(B(u, \bar{u}) \mid \xi)} e^{\left(\xi^{\frac{1}{2}} \circ u \mid u_{0}\right)} e^{-\left(u_{0} \mid u_{0}\right)} \\
& \quad \times \pi\left(r(2 t)^{\frac{1}{2}} k(-i u) r\left(\xi^{\frac{1}{2}}\right) k\left(i u_{0}\right)\right)^{*^{-1}} \phi\left(\xi, u_{0}\right) d u_{0} \|^{2} d \xi \\
& =2^{n_{1}} \pi^{-r b} \int_{\Omega} \| \int_{V_{1}} e^{-(t \mid \xi)} e^{-\frac{1}{2}(B(u, \bar{u}) \mid \xi)} e^{\left(\xi^{\frac{1}{2}} \circ u \mid u_{0}\right)} e^{-\left(u_{0} \mid u_{0}\right)} \\
& \quad \pi\left(r(2 t)^{\frac{1}{2}} r\left(\xi^{\frac{1}{2}}\right) k\left(-i \xi^{\frac{1}{2}} \circ u\right) k\left(i u_{0}\right)\right)^{*}{ }^{-1} \phi\left(\xi, u_{0}\right) d u_{0} \|^{2} d \xi .
\end{aligned}
$$

Then

$$
\begin{aligned}
& \int_{\Sigma}\left\|\pi\left(r(2 y-B(u, \bar{u}))^{\frac{1}{2}} k(-i u)\right)^{*^{-1}} \Phi(x+i y, u)\right\|^{2} \Delta(2 y-B(u, \bar{u}))^{-\mu+\alpha} d x d y d u \\
& =2^{n_{1}} \pi^{-r b} \int_{\Omega} \int_{\Omega} \int_{V_{1}} e^{-2(t \mid \xi)} \Delta(2 t)^{-\mu+\alpha} \| \int_{V_{1}} e^{-\frac{1}{2}(B(u, \bar{u}) \mid \xi)} e^{\left(\xi^{\frac{1}{2}} \circ u \mid u_{0}\right)} \\
& \quad \times e^{-\left(u_{0} \mid u_{0}\right)}\left(\pi(2 t)^{\frac{1}{2}} \pi\left(\xi^{\frac{1}{2}}\right) \pi\left(k\left(i\left(u_{0}-\xi^{\frac{1}{2}} \circ u\right)\right)\right)\right)^{*-1} \phi\left(\xi, u_{0}\right) d u_{0} \|^{2} d \xi d t d u .
\end{aligned}
$$

By (4.4.3) and (2.7.4),

$$
\left(\xi^{\frac{1}{2}} \circ u \mid \xi^{\frac{1}{2}} \circ u\right)=\left(B\left(\xi^{\frac{1}{2}} \circ u, \overline{\xi^{\frac{1}{2}} \circ u}\right) \mid e\right)=\left(P\left(\xi^{\frac{1}{2}}\right) B(u, \bar{u}) \mid e\right)=(B(u, \bar{u}) \mid \xi) .
$$

By a change of variables and [Di2], Theorem 4.3, we have

(4)

$$
\begin{aligned}
& \int_{\Sigma}\left\|\pi\left(r(2 y-B(u, \bar{u}))^{\frac{1}{2}} k(-i u)\right)^{*^{-1}} \Phi(x+i y, u)\right\|^{2} \Delta(2 y-B(u, \bar{u}))^{-\mu+\alpha} d x d y d u \\
& =2^{n_{1}} \int_{\Omega \oplus V_{1}} \operatorname{tr}\left[\phi ( \xi , u _ { 0 } ) ^ { * } \left(\int_{\Omega \oplus V_{1}} e^{-2(t \mid \xi)} e^{-\left(\xi^{\frac{1}{2}} \circ u \mid \xi^{\frac{1}{2}} \circ u\right)}\right.\right. \\
& \times\left(\pi(2 t)^{\frac{1}{2}} \pi\left(\xi^{\frac{1}{2}}\right) \pi\left(k\left(i \xi^{\frac{1}{2}} \circ u\right)\right)\right)^{-1}\left(\pi(2 t)^{\frac{1}{2}} \pi\left(\xi^{\frac{1}{2}}\right) \pi\left(k\left(i \xi^{\frac{1}{2}} \circ u\right)\right)\right)^{*-1} \\
& \left.\left.\times \Delta(2 t)^{-\mu+\alpha} d t d u\right) \phi\left(\xi, u_{0}\right)\right] e^{-\left(u_{0} \mid u_{0}\right)} d \xi d u_{0} .
\end{aligned}
$$

By (3.10.1), (3.10.2), (4.3.3) and variable changes, we obtain that

$$
\begin{aligned}
& \int_{\Omega \oplus V_{1}} e^{-2(t \mid \xi)} e^{-\left(\xi^{\frac{1}{2}} \circ u \mid \xi^{\frac{1}{2}} \circ u\right)}\left(\pi(2 t)^{\frac{1}{2}} \pi\left(\xi^{\frac{1}{2}}\right) \pi\left(k\left(i \xi^{\frac{1}{2}} \circ u\right)\right)\right)^{-1} \\
& \times\left(\pi(2 t)^{\frac{1}{2}} \pi\left(\xi^{\frac{1}{2}}\right) \pi\left(k\left(i \xi^{\frac{1}{2}} \circ u\right)\right)\right)^{*-1} \Delta(2 t)^{-\mu+\alpha} d t d u \\
& =2^{-n_{1}} \Delta(\xi)^{-\alpha-b} \Gamma_{\pi}(\alpha) .
\end{aligned}
$$

Now (1) follows directly from (4) and (5). 
4.9. Corollary. Let $\pi, \mathcal{E}$ be given in Section 4.1, and let $\alpha$ be a real number such that $\omega(\pi)>\mu-\alpha-1$. Suppose that $\phi: A K_{-\frac{1}{2}} \rightarrow \mathcal{E}$ is a measurable function such that it is holomorphic in $u_{0}$, it satisfies the $\pi$-covariant condition

$$
\phi\left(a k\left(u_{0}\right) l\right)=\pi(l)^{-1} \phi\left(a k\left(u_{0}\right)\right)
$$

for $a \in A, u_{0} \in V_{1}, l \in L$, and the integral

$$
\begin{aligned}
\Phi(z, u)=\pi^{-\frac{n}{2}} & \int_{A K_{-\frac{1}{2}}} e^{i(z \mid a e)} e^{\left(u \mid a u_{0}\right)} e^{-\left(u_{0} \mid u_{0}\right)} \\
& \times \pi\left(a k\left(i u_{0}\right)\right)^{*-1} \phi\left(a k\left(u_{0}\right)\right) \Delta(a e)^{\mu} d a d u_{0}
\end{aligned}
$$

is convergent absolutely for all $(z, u) \in \Sigma$. Then $\Phi$ is holomorphic in $\Sigma$ and

$$
\begin{aligned}
\int_{\Sigma} \| & \pi\left(r(2 y-B(u, \bar{u}))^{\frac{1}{2}} k(-i u)\right)^{*-1} \Phi(x+i y, u) \|^{2} \Delta(2 y-B(u, \bar{u}))^{-\mu+\alpha} d x d y d u \\
= & \int_{A K_{-\frac{1}{2}}}\left(\Gamma_{\pi}(\alpha) \phi\left(a k\left(u_{0}\right)\right) \mid \phi\left(a k\left(u_{0}\right)\right) \Delta(a e)^{\mu-\alpha-b} e^{-\left(u_{0} \mid u_{0}\right)} d a d u_{0} .\right.
\end{aligned}
$$

(Note that we do not assert the finiteness of the two integrals in (3); however, if one integral is infinite, then so is the other. The same is true for (4.8.1). Note that Theorem 4.8 and Corollary 4.9 are also valid for $\mathcal{V}_{\pi}$-valued functions $\phi$ and $\Phi$.

Proof. The Corollary follows from Theorem 4.8, (3.10.1) and (3.10.2).

\section{Operator-VAlued Bessel functions}

In this section, we define the operator-valued Bessel function by the inverse Laplace transform, and study its square-integrability with respect to certain measures by Theorem 4.8 .

5.1. Inversion formula on the cone. Let $\phi: \Omega \rightarrow \mathcal{E}$ be a measurable function on the cone $\Omega$ such that

$$
\Phi(x+i y)=(2 \pi)^{-\frac{n_{1}}{2}} \int_{\Omega} e^{i(x+i y \mid \xi)} \pi\left(\xi^{\frac{1}{2}}\right)^{-1} \phi(\xi) d \xi
$$

converges absolutely for all $x+i y \in J+i \Omega$. We call $\Phi$ the $\pi$-weighted Laplace transform of $\phi$. The Laplace inversion formula

$$
\phi(\xi)=(2 \pi)^{-\frac{n_{1}}{2}} \int_{J} e^{-i(x+i y \mid \xi)} \pi\left(\xi^{\frac{1}{2}}\right) \Phi(x+i y) d x
$$

is valid whenever the function $x \mapsto \Phi(x+i y)$ is integrable. Conversely, suppose $\Phi: J+i \Omega \rightarrow \mathcal{E}$ is holomorphic, the integral

$$
I_{\Phi}(y)=\int_{J}\|\Phi(x+i y)\| d x
$$

exists for all $y \in \Omega$, and as a function of $y$ the integral $I_{\Phi}$ is bounded on any compact subset of $\Omega$. Then there exists a continuous function $\phi$ on $J$ such that $\Phi$ is the $\pi$-weighted Laplace transform of $\phi$ defined by (1); and if for any $\epsilon>0$ the function $y \mapsto I_{\Phi}(y) e^{-\epsilon|| y \|}$ is bounded as $\|y\| \rightarrow \infty$, then $\phi(\xi)=0$ for all $\xi$ not in $\Omega$. In fact, $\phi$ is determined by (2) (see [B2]). 
5.2. Lemma. If $\omega(\pi)>2 \mu-1$, then

$$
\beta=\int_{J}\left[\operatorname{tr}\left(\pi\left(x^{2}+e\right)\right)\right]^{1 / 2} d x
$$

is finite ([DG1], Lemma 3.3), and

$$
\int_{J}\|\pi(x+i y)\| d x \leq \Delta(y)^{\mu}\left\|\pi\left(y^{\frac{1}{2}}\right)\right\|^{2} \beta
$$

for $y \in \Omega$ ([DG1], Section 3.4).

5.3. Theorem. Suppose that $\omega(\pi)>2 \mu-1$. For fixed $v_{0} \in V_{1}$ the integral

$$
\begin{aligned}
& 2^{-n_{1}} \pi^{-\left(\frac{n}{2}+r b\right)} \int_{J \oplus V_{1}} e^{-i\left(x+i\left(t+\frac{1}{2} B(u, \bar{u})\right) \mid \xi\right)} e^{\left(u_{0} \mid \xi^{\frac{1}{2}} \circ u\right)} e^{-\left(\xi^{\frac{1}{2}} \circ u \mid \xi^{\frac{1}{2}} \circ u\right)} \\
& \times e^{-i\left(\left(x+i\left(t+\frac{1}{2} B(u, \bar{u})\right)\right)^{-1} \mid e\right)} e^{\left(\left(x+i\left(t+\frac{1}{2} B(u, \bar{u})\right)\right)^{-1} \mid B\left(v_{0}, \bar{u}\right)\right)} \Delta(\xi)^{b} \\
& \times \pi\left(r\left(\xi^{\frac{1}{2}}\right) k\left(i \xi^{\frac{1}{2}} \circ u\right)\right) \pi\left(\left(x+i\left(t+\frac{1}{2} B(u, \bar{u})\right)\right) \pi(k(u)) \pi\left(k\left(-i v_{0}\right)\right)^{*} d x d u\right.
\end{aligned}
$$

converges absolutely, is independent of the choice of $t \in \Omega$, and defines an $\mathcal{E}$-valued continuous function $\phi\left(\xi, u_{0}\right)$ on $\Omega \oplus V_{1}$. Moreover, $\phi$ is holomorphic in $u_{0}$ and the $\pi$-weighted Laplace transform $\Phi$ of $\phi$ defined by (4.6.1) is

$$
\Phi(z, u)=e^{-i\left(z^{-1} \mid e\right)} e^{\left(z^{-1} \mid B\left(v_{0}, \bar{u}\right)\right)} \pi(z) \pi(k(u)) \pi\left(k\left(-i v_{0}\right)\right)^{*},
$$

where $(z, u) \in \Sigma$.

Proof. For $y \in \Omega, \quad \operatorname{Im}\left[(x+i y)^{-1}\right]<0$. Then $\operatorname{Re}\left[-i\left((x+i y)^{-1} \mid e\right)\right]<0$ and $\left|e^{\left.-i(x+i y)^{-1} \mid e\right)}\right|<1$. Since $B(\cdot, \cdot)$ is positive definite, $B\left(u-v_{0}, \overline{u-v_{0}}\right) \geq 0$ and $B\left(u+v_{0}, \overline{u+v_{0}}\right) \geq 0$. Hence,

$$
-\frac{1}{2}\left[B(u, \bar{u})+B\left(v_{0}, \bar{v}_{0}\right)\right] \leq \operatorname{Re} B\left(v_{0}, \bar{u}\right) \leq \frac{1}{2}\left[B(u, \bar{u})+B\left(v_{0}, \bar{v}_{0}\right)\right] .
$$

Replacing $v_{0}$ by $i v_{0}$, we have

$$
-\frac{1}{2}\left[B(u, \bar{u})+B\left(v_{0}, \bar{v}_{0}\right)\right] \leq \operatorname{Im} B\left(v_{0}, \bar{u}\right) \leq \frac{1}{2}\left[B(u, \bar{u})+B\left(v_{0}, \bar{v}_{0}\right)\right] .
$$

Thus,

$$
\begin{aligned}
& \left|\left(\left(x+i\left(t+\frac{1}{2} B(u, \bar{u})\right)\right)^{-1} \mid B\left(v_{0}, \bar{u}\right)\right)\right| \\
& \leq\left|\left(\left(x+i\left(t+\frac{1}{2} B(u, \bar{u})\right)\right)^{-1} \mid B(u, \bar{u})\right)\right|+\left|\left(\left(x+i\left(t+\frac{1}{2} B(u, \bar{u})\right)\right)^{-1} \mid B\left(v_{0}, \bar{v}_{0}\right)\right)\right| .
\end{aligned}
$$

$\left|\left(\left(x+i\left(t+\frac{1}{2} B(u, \bar{u})\right)\right)^{-1} \mid B\left(v_{0}, \bar{u}\right)\right)\right|$ and $\left|e^{\left(\left(x+i\left(t+\frac{1}{2} B(u, \bar{u})\right)\right)^{-1} \mid B\left(v_{0}, \bar{u}\right)\right)}\right|$ are bounded for fixed $t \in \Omega$ and $v_{0} \in V_{1}$. By Section 5.1 and Lemma 5.2, the integral

$$
\begin{aligned}
& \int_{J} e^{-i\left(x+i\left(t+\frac{1}{2} B(u, \bar{u})\right) \mid \xi\right)} e^{-i\left(\left(x+i\left(t+\frac{1}{2} B(u, \bar{u})\right)\right)^{-1} \mid e\right)} \\
& \times e^{\left(\left(x+i\left(t+\frac{1}{2} B(u, \bar{u})\right)\right)^{-1} \mid B\left(v_{0}, \bar{u}\right)\right)} \pi\left(x+i\left(t+\frac{1}{2} B(u, \bar{u})\right)\right) d x
\end{aligned}
$$

converges absolutely and the integral vanishes for all $\xi$ not in $\Omega$. Since $\pi(k(u))$ is a polynomial in $u$ (cf. [RV1], Proposition 5.6(b)), the integral (1) also converges absolutely, and it defines an $\mathcal{E}$-valued function $\phi\left(\xi, u_{0}\right)$. 
By (1), (4.6.1) and (4.7.4)

$$
\begin{gathered}
\Phi(z, u)=2^{-n_{1}} \pi^{-(n+r b)} \int_{\Omega \oplus V_{1}} e^{i(z \mid \xi)} e^{\left(\xi^{\frac{1}{2}} \circ u \mid u_{0}\right)} e^{-\left(u_{0} \mid u_{0}\right)} \pi\left(r\left(\xi^{\frac{1}{2}}\right) k\left(i u_{0}\right)\right)^{*} \\
\times\left[\int_{J \oplus V_{1}} e^{-i\left(x^{\prime}+i y^{\prime} \mid \xi\right)} e^{\left(u_{0} \mid \xi^{\frac{1}{2}} \circ u^{\prime}\right)} e^{-\left(\xi^{\frac{1}{2}} \circ u^{\prime} \mid \xi^{\frac{1}{2}} \circ u^{\prime}\right)} e^{-i\left(\left(x^{\prime}+i y^{\prime}\right)^{-1} \mid e\right)} e^{\left(\left(x^{\prime}+i y^{\prime}\right)^{-1} \mid B\left(v_{0}, \bar{u}^{\prime}\right)\right)}\right. \\
\left.\times \Delta(\xi)^{b} \pi\left(r\left(\xi^{\frac{1}{2}}\right) k\left(i \xi^{\frac{1}{2}} \circ u^{\prime}\right)\right)^{*} \pi\left(x^{\prime}+i y^{\prime}\right) \pi\left(k\left(u^{\prime}\right)\right) \pi\left(k\left(-i v_{0}\right)\right)^{*} d x^{\prime} d u^{\prime}\right] d \xi d u_{0} \\
=2^{-n_{1}} \pi^{-n} \int_{\Omega} e^{i(z \mid \xi)}\left[\int_{J \oplus V_{1}} e^{\left(\xi^{\frac{1}{2}} \circ u \mid \xi^{\frac{1}{2}} \circ u^{\prime}\right)} e^{-i\left(x^{\prime}+i y^{\prime} \mid \xi\right)} e^{-\left(\xi^{\frac{1}{2}} \circ u^{\prime} \mid \xi^{\frac{1}{2}} \circ u^{\prime}\right)}\right. \\
\times e^{-i\left(\left(x^{\prime}+i y^{\prime}\right)^{-1} \mid e\right)} e^{\left(\left(x^{\prime}+i y^{\prime}\right)^{-1} \mid B\left(v_{0}, \bar{u}^{\prime}\right)\right)} \Delta(\xi)^{b} \pi\left(r\left(\xi^{\frac{1}{2}}\right) k\left(i \xi^{\frac{1}{2}} \circ u\right)\right)^{*}-1 \\
\left.\times \pi\left(r\left(\xi^{\frac{1}{2}}\right) k\left(i \xi^{\frac{1}{2}} \circ u^{\prime}\right)\right)^{*} \pi\left(x^{\prime}+i y^{\prime}\right) \pi\left(k\left(u^{\prime}\right)\right) \pi\left(k\left(-i v_{0}\right)\right)^{*} d x^{\prime} d u^{\prime}\right] d \xi \\
=2^{-n_{1}} \pi^{-n_{1}} \int_{\Omega} e^{i(z \mid \xi)}\left[\int_{J} e^{-i\left(x^{\prime}+i y^{\prime} \mid \xi\right)} e^{-i\left(\left(x^{\prime}+i y^{\prime}\right)^{-1} \mid e\right)}\right. \\
\left.\times e^{\left(\left(x^{\prime}+i y^{\prime}\right)^{-1} \mid B\left(v_{0}, \bar{u}\right)\right)} \pi\left(x^{\prime}+i y^{\prime}\right) \pi(k(u)) \pi\left(k\left(-i v_{0}\right)\right)^{*} d x^{\prime}\right] d \xi .
\end{gathered}
$$

Then (2) follows from Section 5.1 and Lemma 5.2.

We now define the operator-valued Bessel function $K_{\pi}$ by the inverse Laplace transform.

5.4. Definition. By Theorem 5.3, there is an $\mathcal{E}$-valued function $K_{\pi}\left(\xi, u_{0} ; v_{0}\right)$ on $\Omega \oplus V_{1} \oplus V_{1}$ such that

$$
\int_{\Omega \oplus V_{1}} e^{i(z \mid \xi)} e^{\left(\xi^{\frac{1}{2}} \circ u \mid u_{0}\right)} e^{-\left(u_{0} \mid u_{0}\right)} \pi\left(r\left(\xi^{\frac{1}{2}}\right) k\left(i u_{0}\right)\right)^{*} K_{\pi}\left(\xi, u_{0} ; v_{0}\right) \Delta(\xi)^{-\mu} d \xi d u_{0}
$$

$$
=e^{-i\left(z^{-1} \mid e\right)} e^{\left(z^{-1} \mid B\left(v_{0}, \bar{u}\right)\right)} \pi(z) \pi(k(u)) \pi\left(k\left(-i v_{0}\right)\right)^{*} .
$$

We call $K_{\pi}$ the (operator-valued) Bessel function of weight $\pi$.

By polar coordinates, we can define $K_{\pi}\left(a k\left(u_{0}\right) ; v_{0}\right)$ on $\left(A K_{-\frac{1}{2}}\right) \times V_{1}$. By $(3.10 .1)$ and (3.10.2), we have

$$
\begin{aligned}
& \int_{A K_{-\frac{1}{2}}} e^{i(z \mid a e)} e^{\left(u \mid a u_{0}\right)} e^{-\left(u_{0} \mid u_{0}\right)} \pi\left(a k\left(i u_{0}\right)\right)^{*-1} K_{\pi}\left(a k\left(u_{0}\right) ; v_{0}\right) d a d u_{0} \\
& =e^{-i\left(z^{-1} \mid e\right)} e^{\left(z^{-1} \mid B\left(v_{0}, \bar{u}\right)\right)} \pi(z) \pi(k(u)) \pi\left(k\left(-i v_{0}\right)\right)^{*} .
\end{aligned}
$$

5.5. Lemma. For $u \in V_{1}$ and invertible $z \in V_{2}$, we have

$$
\begin{aligned}
& k(-u) r(z)^{-1} k(-i u)^{*} r(2 \operatorname{Im} z-B(u, \bar{u})) k(-i u) r\left(z^{*}\right)^{-1} k(-u)^{*} \\
& =k\left(-i z^{-1} \circ u\right)^{*} r\left(2 \operatorname{Im}\left(-z^{-1}\right)-B\left(z^{-1} \circ u, \overline{z^{-1} \circ u}\right)\right) k\left(-i z^{-1} \circ u\right) .
\end{aligned}
$$


Proof. By (3.9.1), (3.9.2) and (3.8.5), we have

(2)

$$
\begin{aligned}
& k(-u) r\left(z^{-1}\right) k(-i u)^{*} r(2 \operatorname{Im} z-B(u, \bar{u})) k(-i u) r\left(z^{*}\right)^{-1} k(-u)^{*} \cdot(w, v) \\
& =\left(P(z)^{-1} P(2 \operatorname{Im} z-B(u, \bar{u})) P\left(z^{*}\right)^{-1} w\right. \\
& +P(z)^{-1} P(2 \operatorname{Im} z-B(u, \bar{u})) P\left(z^{*}\right)^{-1} B(v,-\bar{u}) \\
& +P(z)^{-1} B\left(( 2 \operatorname { I m } z - B ( u , \overline { u } ) ) \circ \left\{\left[P\left(z^{*}\right)^{-1} w+P\left(z^{*}\right)^{-1} B(v,-\bar{u})\right] \circ(-i u)\right.\right. \\
& \left.\left.+z^{*-1} \circ v\right\}, i \bar{u}\right), \quad\left\{P(z)^{-1} P(2 \operatorname{Im} z-B(u, \bar{u})) P\left(z^{*}\right)^{-1} w\right. \\
& +P(z)^{-1} P(2 \operatorname{Im} z-B(u, \bar{u})) P\left(z^{*}\right)^{-1} B(v,-\bar{u})+P(z)^{-1} B((2 \operatorname{Im} z-B(u, \bar{u})) \\
& \left.\left.\circ\left(\left[P\left(z^{*}\right)^{-1} w+P\left(z^{*}\right)^{-1} B(v,-\bar{u})\right] \circ(-i u)+z^{*-1} \circ v\right), i \bar{u}\right)\right\} \circ(-u) \\
& \left.+z^{-1} \circ\left\{(2 \operatorname{Im} z-B(u, \bar{u})) \circ\left(\left[P\left(z^{*}\right)^{-1} w+P\left(z^{*}\right)^{-1} B(v, \bar{u})\right] \circ(-i u)+z^{*-1} \circ v\right)\right\}\right),
\end{aligned}
$$

and

(3)

$$
\begin{aligned}
k\left(-i z^{-1} \circ u\right)^{*} r\left(2 \operatorname{Im}\left(-z^{-1}\right)-B\left(z^{-1} \circ u, \overline{z^{-1} \circ u}\right)\right) k\left(-i z^{-1} \circ u\right) \cdot(w, v) \\
=\left(P\left(-2 \operatorname{Im}\left(z^{-1}\right)-B\left(z^{-1} \circ u, \overline{z^{-1} \circ u}\right)\right) w\right. \\
+B\left(\left(-2 \operatorname{Im}\left(z^{-1}\right)-B\left(z^{-1} \circ u, \overline{z^{-1} \circ u}\right)\right) \circ\left(w \circ\left(-i z^{-1} \circ u\right)+v\right), \overline{-i z^{-1} \circ u}\right), \\
\left.\quad\left(-2 \operatorname{Im}\left(z^{-1}\right)-B\left(z^{-1} \circ u, \overline{z^{-1} \circ u}\right)\right)\left(w \circ\left(-i z^{-1} \circ u\right)+v\right)\right) .
\end{aligned}
$$

for $(w, v) \in \Sigma$.

By (2.2.1), (2.7.5), (2.7.6) and a calculation, we have

$$
\begin{gathered}
P(z)^{-1} P(2 \operatorname{Im} z-B(u, \bar{u})) P\left(z^{*}\right)^{-1} w \\
=P(z)^{-1}\left\{[P(2 \operatorname{Im} z)+P(B(u, \bar{u}))] P\left(z^{*}\right)^{-1} w-B\left((2 \operatorname{Im} z) \circ\left(P\left(z^{*}\right)^{-1} w \circ u\right), \bar{u}\right)\right. \\
\left.-B\left(u, \overline{(2 \operatorname{Im} z) \circ\left(\left(P\left(z^{*}\right)^{-1} w\right)^{*} \circ u\right)}\right)\right\} \\
P\left(-2 \operatorname{Im}\left(z^{-1}\right)-B\left(z^{-1} \circ u, \overline{z^{-1} \circ u}\right)\right) w \\
=P\left(-2 \operatorname{Im}\left(z^{-1}\right)\right) w+P\left(B\left(z^{-1} \circ u, \overline{z^{-1} \circ u}\right)\right) w \\
-B\left(-2 \operatorname{Im}\left(z^{-1}\right) \circ\left(w \circ\left(z^{-1} \circ u\right)\right), \overline{z^{-1} \circ u}\right) \\
-B\left(z^{-1} \circ u, \overline{-2 \operatorname{Im}\left(z^{-1}\right) \circ\left(w^{*} \circ\left(z^{-1} \circ u\right)\right)}\right), \\
P(z)^{-1} P(B(u, \bar{u})) P\left(z^{*}\right)^{-1} w \\
=\frac{1}{2} P(z)^{-1}\left\{B\left(B(u, \bar{u}) \circ\left(P\left(z^{*}\right)^{-1} w \circ u\right), \bar{u}\right)\right. \\
\left.+B\left(u, \overline{B(u, \bar{u})^{*} \circ\left(\left(P\left(z^{*}\right)^{-1} w\right)^{*} \circ u\right)}\right)\right\}
\end{gathered}
$$

and

$$
\begin{aligned}
& P\left(B\left(z^{-1} \circ u, \overline{z^{-1} \circ u}\right)\right) w=\frac{1}{2}\left\{B\left(B\left(z^{-1} \circ u, \overline{z^{-1} \circ u}\right) \circ\left(w \circ\left(z^{-1} \circ u\right)\right), \overline{z^{-1} \circ u}\right)\right. \\
& \left.+B\left(z^{-1} \circ u, \overline{B\left(z^{-1} \circ u, \overline{z^{-1} \circ u}\right)^{*} \circ\left(w^{*} \circ\left(z^{-1} \circ u\right)\right)}\right)\right\} .
\end{aligned}
$$


By [Do2], (4.2) and [Do1], Theorem 3.3,

$$
B\left(B(u, \bar{u}) \circ\left(P\left(z^{*}\right)^{-1} w \circ u\right), \bar{u}\right)=B\left(u, \overline{\left.B(u, \bar{u})^{*} \circ\left(\left(P\left(z^{*}\right)^{-1} w\right)^{*} \circ u\right)\right)}\right)
$$

and

$$
\begin{aligned}
& B\left(B\left(z^{-1} \circ u, \overline{z^{-1} \circ u}\right) \circ\left(w \circ\left(z^{-1} \circ u\right)\right), \overline{z^{-1} \circ u}\right) \\
& =B\left(z^{-1} \circ u, \overline{B\left(z^{-1} \circ u, \overline{z^{-1} \circ u}\right)^{*} \circ\left(w^{*} \circ\left(z^{-1} \circ u\right)\right)}\right) .
\end{aligned}
$$

By (2.2.4), (2.7.4) and (2.7.6), we have

$$
\begin{aligned}
& P(z)^{-1} P(2 \operatorname{Im} z-B(u, \bar{u})) P\left(z^{*}\right)^{-1} w \\
& \quad+P(z)^{-1} B\left((2 \operatorname{Im} z-B(u, \bar{u})) \circ\left[P\left(z^{*}\right)^{-1} w \circ u\right], \bar{u}\right) \\
& =P(z)^{-1} P(2 \operatorname{Im} z) P\left(z^{*}\right)^{-1} w-P(z)^{-1} B\left(u, \overline{(2 \operatorname{Im} z) \circ\left(\left(P\left(z^{*}\right)^{-1} w\right)^{*} \circ u\right)}\right) \\
& =P\left(-2 \operatorname{Im}\left(z^{-1}\right)\right) w-B\left(z^{-1} \circ u, \overline{\left(-2 \operatorname{Im}\left(z^{-1}\right)\right) \circ\left(w^{*} \circ\left(z^{-1} \circ u\right)\right)}\right) \\
& =P\left(-2 \operatorname{Im}\left(z^{-1}\right)-B\left(z^{-1} \circ u, \overline{\left.z^{-1} \circ u\right)}\right) w\right. \\
& +B\left(\left(-2 \operatorname{Im}\left(z^{-1}\right)-B\left(z^{-1} \circ u, \overline{z^{-1} \circ u}\right)\right) \circ\left(w \circ\left(z^{-1} \circ u\right)\right), \overline{z^{-1} \circ u}\right) .
\end{aligned}
$$

By similar calculations, we can show that the right sides of (2) and (3) are equal. Hence,

$$
\begin{aligned}
& k(-u) r\left(z^{-1}\right) k(-i u)^{*} r(2 \operatorname{Im} z-B(u, \bar{u})) k(-i u) r\left(z^{*}\right)^{-1} k(-u)^{*} \cdot(w, v) \\
& =k\left(-i z^{-1} \circ u\right)^{*} r\left(2 \operatorname{Im}\left(-z^{-1}\right)-B\left(z^{-1} \circ u, \overline{z^{-1} \circ u}\right)\right) k\left(-i z^{-1} \circ u\right) \cdot(w, v)
\end{aligned}
$$

for $(w, v) \in \Sigma$, and the lemma is proved.

We next apply Theorem 4.8 to obtain the square-integrability of $K_{\pi}$ with respect to certain measures on $\Omega \oplus V_{1} \oplus V_{1}$.

5.6. Theorem. Let $\alpha$ be a real number such that $\alpha+\omega(\pi)>\mu-1$. Then

$$
\begin{aligned}
& \int_{\Omega \oplus V_{1} \oplus V_{1}}\left(\Gamma_{\pi}(\alpha) K_{\pi}\left(\xi, u ; v_{0}\right) \mid K_{\pi}\left(\xi, u ; v_{0}\right)\right) \Delta(\xi)^{-2 \mu-\alpha-b} e^{-(u \mid u)} e^{-\left(v_{0} \mid v_{0}\right)} d \xi d u d v_{0} \\
& =c \operatorname{tr} \int_{\Omega \oplus V_{1}} e^{-2(t \mid e)} e^{-(u \mid u)} \pi(k(i u)) \pi(2 t)^{-1} \pi(k(i u))^{*} \Delta(2 t)^{-2 \mu-\alpha-b} d t d u \\
& \quad \times \int_{J \oplus V_{1}} \Delta\left(x+i\left(e+\frac{1}{2} B(u, \bar{u})\right)\right)^{-2(\mu+b+\alpha)} d x d u
\end{aligned}
$$

for some constant $c$. The integrals in (1) are finite for $\alpha$ in the interval

$$
-\frac{1}{2}<\alpha<\omega(\pi)-2 \mu-b+1
$$

Proof. By Theorem 4.4, $\Gamma_{\pi}(\alpha)$ is well defined. We apply Theorem 4.8 with

$$
\phi(\xi, u)=\pi^{\frac{n}{2}} K_{\pi}\left(\xi, u ; v_{0}\right) \Delta(\xi)^{-\mu} .
$$


By (5.4.1), (4.8.1), (4.6.1) and (4.1.1), we have

$$
\begin{aligned}
& \int_{\Omega \oplus V_{1}}\left(\Gamma_{\pi}(\alpha) K_{\pi}\left(\xi, u ; v_{0}\right) \mid K_{\pi}\left(\xi, u ; v_{0}\right)\right) \Delta(\xi)^{-2 \mu-\alpha-b} e^{-(u \mid u)} e^{-\left(v_{0} \mid v_{0}\right)} d \xi d u \\
& =\pi^{-n} \int_{\Sigma}|| \pi\left(r(2 y-B(u, \bar{u}))^{\frac{1}{2}} k(-i u)\right)^{*^{-1}} e^{-i\left(z^{-1} \mid e\right)} e^{\left(z^{-1} \mid B\left(v_{0}, \bar{u}\right)\right)} \pi(z) \\
& \times\left.\pi(k(u)) \pi\left(k\left(-i v_{0}\right)\right)^{*}\right|^{2} \Delta(2 y-B(u, \bar{u}))^{-\mu+\alpha} e^{-\left(v_{0} \mid v_{0}\right)} d x d y d u \\
& =\pi^{-n} \int_{\Sigma}\left|e^{-i\left(z^{-1} \mid e\right)}\right|^{2} e^{2 \operatorname{Re}\left(z^{-1} \mid B\left(v_{0}, \bar{u}\right)\right)} e^{-\left(v_{0} \mid v_{0}\right)} \\
& \times \operatorname{tr}\left\{\pi\left(k\left(-i v_{0}\right)\right) \pi(k(u))^{*} \pi(z)^{*} \pi(k(i u)) \pi(2 y-B(u, \bar{u}))^{-1} \pi(k(i u))^{*}\right. \\
& \left.\times \pi(z) \pi(k(u)) \pi\left(k\left(-i v_{0}\right)\right)^{*}\right\} \Delta(2 y-B(u, \bar{u}))^{-\mu+\alpha} d x d y d u
\end{aligned}
$$

By (5.5.1), we have

$$
\begin{aligned}
& k(u)^{*} r\left(z^{*}\right) k(i u) r(2 \operatorname{Im} z-B(u, \bar{u}))^{-1} k(i u)^{*} r(z) k(u) \\
& =k\left(i z^{-1} \circ u\right) r\left(2 \operatorname{Im}\left(-z^{-1}\right)-B\left(z^{-1} \circ u, \overline{z^{-1} \circ u}\right)\right)^{-1} k\left(i z^{-1} \circ u\right)^{*} .
\end{aligned}
$$

By [Gi], (2.50), $\Delta(2 y-B(u, \bar{u}))^{-2 \mu-b} d x d y d u$ is an invariant measure under the variable changes $z \mapsto-z^{-1}, u \mapsto z \circ u$. Then (3) is equal to

$$
\begin{aligned}
& \pi^{-n} \int_{\Sigma} e^{-2(y \mid e)} e^{2 \operatorname{Re}\left(e \mid B\left(v_{0} \mid \bar{u}\right)\right)} e^{-\left(v_{0} \mid v_{0}\right)} \operatorname{tr}\left\{\pi\left(k\left(-i v_{0}\right)\right) \pi(k(i u)) \pi(2 y-B(u, \bar{u}))^{-1}\right. \\
& \left.\quad \times \pi(k(i u))^{*} \pi\left(k\left(-i v_{0}\right)\right)^{*}\right\} \Delta(2 y-B(u, \bar{u}))^{-\mu+\alpha} \Delta(z)^{-2(\mu+b+\alpha)} d x d y d u \\
& =\pi^{-n} \operatorname{tr} \int_{\Sigma} e^{-2(t \mid e)} e^{-\left(u-v_{0} \mid u-v_{0}\right)} \pi\left(k\left(i\left(u-v_{0}\right)\right)\right) \pi(2 t)^{-1} \\
& \quad \times \pi\left(k\left(i\left(u-v_{0}\right)\right)\right)^{*} \Delta(2 t)^{-\mu+\alpha} \Delta(z)^{-2(\mu+b+\alpha)} d x d y d u,
\end{aligned}
$$

where $z=x+i y$ and $t=y-\frac{1}{2} B(u, \bar{u})$. It follows that

$$
\begin{aligned}
& \int_{\Omega \oplus V_{1} \oplus V_{1}}\left(\Gamma_{\pi}(\alpha) K_{\pi}\left(\xi, u ; v_{0}\right) \mid K_{\pi}\left(\xi, u ; v_{0}\right)\right) \Delta(\xi)^{-2 \mu-\alpha-b} e^{-(u \mid u)} e^{-\left(v_{0} \mid v_{0}\right)} d \xi d u d v_{0} \\
& =c \operatorname{tr} \int_{\Omega \oplus V_{1}} e^{-2(t \mid e)} e^{-(u \mid u)} \pi(k(i u)) \pi(2 t)^{-1} \pi(k(i u))^{*} \Delta(2 t)^{-2 \mu-\alpha-b} d t d u \\
& \quad \times \int_{J \oplus V_{1}} \Delta\left(x+i\left(e+\frac{1}{2} B(u, \bar{u})\right)\right)^{-2(\mu+b+\alpha)} d x d u,
\end{aligned}
$$

where $c$ is a constant. By Theorem 4.4, the first integral converges if $\omega(\pi)>$ $2 \mu+\alpha+b-1$. By [Gi, (5.98)], the second integral converges if $\alpha>-\frac{1}{2}$. The theorem is proved.

5.7. Corollary. If $\omega(\pi)>2 \mu+b-1$, then

$$
\int_{\Omega \oplus V_{1} \oplus V_{1}}\left\|K_{\pi}\left(\xi, u ; v_{0}\right)\right\|^{2} \Delta(\xi)^{-2 \mu-b} e^{-(u \mid u)} e^{-\left(v_{0} \mid v_{0}\right)} d \xi d u d v_{0}<\infty,
$$

or

$$
\int_{\left(A K_{-\frac{1}{2}}\right) \times V_{1}}\left\|K_{\pi}\left(a k(u) ; v_{0}\right)\right\|^{2} \Delta(a e)^{-\mu-b} e^{-(u \mid u)} e^{-\left(v_{0} \mid v_{0}\right)} d a d u d v_{0}<\infty
$$


5.8. Remark. For the rest of the paper, we assume that $\pi$ is an ifdh representation of $K^{\mathbb{C}}$ satisfying

$$
\omega(\pi)>2 \mu+b-1
$$

\section{Unitarily EQuivalent Hilbert SPACES}

In this section, we construct Hilbert spaces $\mathfrak{S F}_{\Gamma}$ and $\mathcal{H}_{\pi}$ of vector-valued functions. $\mathfrak{S F}_{\Gamma}$ is a space of $\pi$-covariant measurable functions on the group $A K_{-\frac{1}{2}}$, and $\mathcal{H}_{\pi}$ is a space of holomorphic functions on the Siegel domain $\Sigma$ of type II. We define an intertwining operator $\mathcal{T}$ that establishes their unitary equivalence. We define the Hankel transform $\mathcal{K}_{\pi}$ corresponding to the Bessel function $K_{\pi}$ and we prove that the Hankel transform is a unitary operator of $\mathfrak{S F}_{\Gamma}$ to $\mathcal{H}_{\pi}$. We also study the reproducing kernel property of $\mathcal{H}_{\pi}$.

6.1. The space $\mathfrak{S F}_{\Gamma}$. By Theorem 4.4, the integral

$$
\begin{aligned}
\Gamma_{\pi} & =\Gamma_{\pi}(-\mu-b) \\
& =\int_{\Omega \oplus V_{1}} e^{-(\xi \mid e)} e^{-\left(u_{0} \mid u_{0}\right)} \pi\left(k\left(i u_{0}\right)^{*} r(\xi) k\left(i u_{0}\right)\right)^{-1} \Delta(\xi)^{-2 \mu-b} d \xi d u_{0}
\end{aligned}
$$

converges absolutely and defines a positive definite operator on $\mathcal{V}_{\pi}$. By (4.5.2)

$$
\pi(l) \Gamma_{\pi}=\Gamma_{\pi} \pi(l)
$$

for all $l \in L$.

Let $\mathfrak{S F}_{\pi}\left(A K_{-\frac{1}{2}}\right)$ be the space of all measurable functions $f: A K_{-\frac{1}{2}} \rightarrow \mathcal{V}_{\pi}$ such that

$$
f\left(a k\left(u_{0}\right) l\right)=\pi(l)^{-1} f\left(a k\left(u_{0}\right)\right)
$$

for $a \in A, u_{0} \in V_{1}$ and $l \in L, f$ is holomorphic in $u_{0}$, and

$$
\int_{A K_{-\frac{1}{2}}}\left\|f\left(a k\left(u_{0}\right)\right)\right\|^{2} e^{-\left(u_{0} \mid u_{0}\right)} d a d u_{0}<\infty .
$$

Then $\mathfrak{S F}_{\pi}\left(A K_{-\frac{1}{2}}\right)$ is a Hilbert space with respect to the inner product

$$
\left\langle f_{1} \mid f_{2}\right\rangle=\int_{A K_{-\frac{1}{2}}}\left(\Gamma_{\pi} f_{1}\left(a k\left(u_{0}\right)\right) \mid f_{2}\left(a k\left(u_{0}\right)\right)\right) e^{-\left(u_{0} \mid u_{0}\right)} d a d u_{0}
$$

and denote the resulting Hilbert space by $\mathfrak{S F}_{\Gamma} \cdot \mathfrak{S F}_{\Gamma}$ is a restricted SchrödingerFock space in the sense of [Di1].

By (3.10.1) and (3.10.2), the space $\mathfrak{S F}_{\Gamma}$ can be identified with the space of all measurable functions $f: \Omega \oplus V_{1} \rightarrow \mathcal{V}_{\pi}$ such that $f$ is holomorphic in $V_{1}$, and

$$
\int_{\Omega \oplus V_{1}}\left\|f\left(\xi, u_{0}\right)\right\|^{2} e^{-\left(u_{0} \mid u_{0}\right)} \Delta(\xi)^{-\mu} d \xi d u_{0}<\infty .
$$

By the Laplace transform introduced in Section $4.6, \mathfrak{S F}_{\Gamma}$ is unitarily equivalent to a space of holomorphic functions on $\Sigma$.

6.2. Theorem. For any $f \in \mathfrak{S F}_{\Gamma}$,

$$
F(z, u)=\pi^{-\frac{n}{2}} \int_{A K_{-\frac{1}{2}}} e^{i(z \mid a e)} e^{\left(u \mid a u_{0}\right)} e^{-\left(u_{0} \mid u_{0}\right)} \pi\left(a k\left(i u_{0}\right)\right)^{*-1} f\left(a k\left(u_{0}\right)\right) d a d u_{0}
$$


is absolutely convergent for all $(z, u) \in \Sigma$ and defines a holomorphic function $F$ : $\Sigma \rightarrow \mathcal{V}_{\pi}$. Moreover,

$$
\begin{aligned}
& \int_{\Sigma}\left\|\pi\left(r(2 y-B(u, \bar{u}))^{\frac{1}{2}} k(-i u)\right)^{*^{-1}} F(x+i y, u)\right\|^{2} \Delta(2 y-B(u, \bar{u}))^{-2 \mu-b} d x d y d u \\
& =\int_{A K_{-\frac{1}{2}}}\left(\Gamma_{\pi} f\left(a k\left(u_{0}\right)\right) \mid f\left(a k\left(u_{0}\right)\right)\right) e^{-\left(u_{0} \mid u_{0}\right)} d a d u_{0} .
\end{aligned}
$$

Proof. As in Theorem 4.4, we write $z=x+i\left(t+\frac{1}{2} B(u, \bar{u})\right)$. Then

$$
\begin{aligned}
& \int_{A K_{-\frac{1}{2}}}\left|e^{i(z \mid a e)} e^{\left(u \mid a u_{0}\right)} e^{-\left(u_{0} \mid u_{0}\right)}\right|\left\|\pi\left(a k\left(i u_{0}\right)\right)^{*-1} f\left(a k\left(u_{0}\right)\right)\right\| d a d u_{0} \\
& \leq \int_{A K_{-\frac{1}{2}}} e^{-(t \mid a e)} e^{-\frac{1}{2}\left(u_{0}-a u \mid u_{0}-a u\right)} e^{-\frac{1}{2}\left(u_{0} \mid u_{0}\right)} \\
& \quad \times\left\|\pi\left(a k\left(i u_{0}\right)\right)^{*-1}||\right\| f\left(a k\left(u_{0}\right)\right) \| d a d u_{0} \\
& \leq\left(\int_{A K_{-\frac{1}{2}}} e^{-2(t \mid a e)} e^{-\left(u_{0}-a u \mid u_{0}-a u\right)}\left\|\pi\left(a k\left(i u_{0}\right)\right)^{*-1}\right\|^{2} d a d u_{0}\right)^{\frac{1}{2}} \\
& \quad \times\left(\int_{A K_{-\frac{1}{2}}}\left\|f\left(a k\left(u_{0}\right)\right)\right\|^{2} e^{-\left(u_{0} \mid u_{0}\right)} d a d u_{0}\right)^{\frac{1}{2}} .
\end{aligned}
$$

The first integral on the right side is finite by Theorem 4.4. The second integral is finite by (6.1.3). Thus the integral (1) is absolutely convergent and $F$ is well defined. Applying Corollary 4.9, which is also valid for $\mathcal{V}_{\pi}$-valued functions, with $\alpha=-\mu-b$ and

$$
\phi\left(a k\left(u_{0}\right)\right)=\Delta(a e)^{-\mu} f\left(a k\left(u_{0}\right)\right),
$$

we obtain that $F$ is holomorphic in $\Sigma$ and (2) is valid.

6.3. The space $\mathcal{H}_{\pi}$. Denote by $\mathcal{H}_{\pi}$ the space of all functions $F$ of the integral form (6.2.1) for $f \in \mathfrak{S F}_{\Gamma}$, where $(z, u) \in \Sigma$. By Theorem 6.2, $\mathcal{H}_{\pi}$ is a Hilbert space with the norm given by

$$
\begin{gathered}
\|F\|^{2}=\int_{\Sigma}\left\|\pi\left(r(2 y-B(u, \bar{u}))^{\frac{1}{2}} k(-i u)\right)^{*^{-1}} F(x+i y, u)\right\|^{2} \\
\times \Delta(2 y-B(u, \bar{u}))^{-2 \mu-b} d x d y d u .
\end{gathered}
$$

By (3.10.1) and (3.10.2), $f \in \mathfrak{S F}_{\Gamma}$ is identified with a function, still denoted by $f$, on $\Omega \oplus V_{1}$, and

$$
\begin{aligned}
F(z, u)= & \pi^{-\frac{n}{2}} \int_{\Omega \oplus V_{1}} e^{i(z \mid \xi)} e^{\left(\xi^{\frac{1}{2}} \circ u \mid u_{0}\right)} e^{-\left(u_{0} \mid u_{0}\right)} \pi\left(r\left(\xi^{\frac{1}{2}}\right) k\left(i u_{0}\right)\right)^{*^{-1}} \\
& \times f\left(\xi, u_{0}\right) \Delta(\xi)^{-\mu} d \xi d u_{0} .
\end{aligned}
$$

6.4. Theorem. The mapping $\mathcal{T}: f \mapsto F$ given by (6.2.1) is a unitary map of $\mathfrak{S F}_{\Gamma}$ onto $\mathcal{H}_{\pi}$. Furthermore, $\mathcal{H}_{\pi}$ is the space of all $\mathcal{V}_{\pi}$-valued holomorphic functions $F$ 
on $\Sigma$ satisfying

$$
\begin{aligned}
\int_{\Sigma} \| & \pi\left(r(2 y-B(u, \bar{u}))^{\frac{1}{2}} k(-i u)\right)^{*-1} F(x+i y, u) \|^{2} \\
& \times \Delta(2 y-B(u, \bar{u}))^{-2 \mu-b} d x d y d u<\infty .
\end{aligned}
$$

Proof. That $\mathcal{T}$ is a unitary map follows from Theorem 6.2. Conversely, let $F$ be a $\mathcal{V}_{\pi}$-valued holomorphic function on $\Sigma$ satisfying (1). By [RV1], Theorem 5.11, the integral

$$
\hat{F}(\xi, u)=\int_{J} F(x+i y, u) e^{-i(x+i y \mid \xi)} d x
$$

is defined for almost all $(\xi, u) \in \Omega \oplus V_{1}$ and is independent of $y$. By [RV1], Lemmas 5.13 and 5.14, $\hat{F}(\xi, u)$ is equal to zero for almost all $\xi \notin \Omega, u \in V_{1}$, and

$$
\int_{V_{1}} e^{-\left(\xi^{\frac{1}{2}} \circ u \mid \xi^{\frac{1}{2}} \circ u\right)}\|\hat{F}(\xi, u)\|^{2} d u<\infty
$$

for almost all $\xi \in \Omega$. Thus, the integrals

$$
\int_{V_{1}} e^{\left(u_{0} \mid \xi^{\frac{1}{2}} \circ u\right)} e^{-\left(\xi^{\frac{1}{2}} \circ u \mid \xi^{\frac{1}{2}} \circ u\right)} \pi\left(r\left(\xi^{\frac{1}{2}}\right) k\left(i \xi^{\frac{1}{2}} \circ u\right)\right) \hat{F}(\xi, u) d u
$$

and

$$
\begin{aligned}
& f\left(\xi, u_{0}\right)=2^{-n_{1}} \pi^{-\left(\frac{n}{2}+r b\right)} \int_{J \oplus V_{1}} e^{-i\left(x+i\left(t+\frac{1}{2} B(u, \bar{u})\right) \mid \xi\right)} e^{\left(u_{0} \mid \xi^{\frac{1}{2}} \circ u\right)} e^{-\left(\xi^{\frac{1}{2}} \circ u \mid \xi^{\frac{1}{2}} \circ u\right)} \\
& (2) \times \Delta(\xi)^{b} \pi\left(r\left(\xi^{\frac{1}{2}}\right) k\left(i \xi^{\frac{1}{2}} \circ u\right)\right) F\left(x+i\left(t+\frac{1}{2} B(u, \bar{u})\right), u\right) d x d u
\end{aligned}
$$

exist for almost all $\xi \in \Omega$, and the integral (2) is independent of the choice of $t>0$. Similar to Proposition 4.7, one can see that $\mathcal{T} f$ given by $(6.3 .2)$ is $F$. Thus, $\mathcal{T}$ is an onto mapping.

We now define the Hankel transform.

6.5. The Hankel transform. Let $K_{\pi}$ be the Bessel function defined by (5.4.1). Let $\left(\mathfrak{S F}_{\Gamma}\right)^{0}$ be the space of functions $f \in \mathfrak{S F}_{\Gamma}$ such that

$$
\int_{A K_{-\frac{1}{2}}}\left\|f\left(a k\left(u_{0}\right)\right)\right\|^{2} e^{-\left(u_{0} \mid u_{0}\right)} \Delta(a e)^{\mu+b} d a d u_{0}<\infty .
$$

(1) is equivalent to the condition

$$
\int_{\Omega \oplus V_{1}}\left\|f\left(\xi, u_{0}\right)\right\|^{2} e^{-\left(u_{0} \mid u_{0}\right)} \Delta(\xi)^{b} d \xi d u_{0}<\infty .
$$

Clearly, $\left(\mathfrak{S} \mathfrak{F}_{\Gamma}\right)^{0}$ is dense in $\mathfrak{S F}_{\Gamma}$. For $f \in\left(\mathfrak{S} \mathfrak{F}_{\Gamma}\right)^{0}$,

$$
\begin{aligned}
& \int_{A K_{-\frac{1}{2}}}\left\|K_{\pi}\left(a_{0}^{*} a k\left(u_{0}\right) ; v_{0}\right) f\left(a_{0} k\left(v_{0}\right)\right)\right\|^{2} e^{-\left(v_{0} \mid v_{0}\right)} d a_{0} d v_{0} \\
& \leq\left(\int_{A K_{-\frac{1}{2}}}\left\|K_{\pi}\left(a_{0}^{*} a k\left(u_{0}\right) ; v_{0}\right)\right\|^{2} \Delta\left(a_{0} e\right)^{-\mu-b} e^{-\left(v_{0} \mid v_{0}\right)} d a_{0} d v_{0}\right)^{\frac{1}{2}} \\
& \times\left(\int_{A K_{-\frac{1}{2}}}\left\|f\left(a_{0} k\left(v_{0}\right)\right)\right\|^{2} \Delta\left(a_{0} e\right)^{\mu+b} e^{-\left(v_{0} \mid v_{0}\right)} d a_{0} d v_{0}\right)^{\frac{1}{2}} \cdot
\end{aligned}
$$


By (5.7.2)

$$
\int_{A K_{-\frac{1}{2}}}\left\|K_{\pi}\left(a_{0}^{*} k\left(u_{0}\right) ; v_{0}\right)\right\|^{2} \Delta\left(a_{0} e\right)^{-\mu-b} e^{-\left(v_{0} \mid v_{0}\right)} d a_{0} d v_{0}<\infty
$$

for almost all $u_{0} \in V_{1}$. By a variable change,

$$
\begin{aligned}
& \left(\int_{A K_{-\frac{1}{2}}}\left\|K_{\pi}\left(a_{0}^{*} a k\left(u_{0}\right) ; v_{0}\right)\right\|^{2} \Delta\left(a_{0} e\right)^{-\mu-b} e^{-\left(v_{0} \mid v_{0}\right)} d a_{0} d v_{0}\right)^{\frac{1}{2}} \\
& \leq\left(\int_{A K_{-\frac{1}{2}}}\left\|K_{\pi}\left(a_{0}^{*} k\left(u_{0}\right) ; v_{0}\right)\right\|^{2} \Delta\left(a_{0} e\right)^{-\mu-b} e^{-\left(v_{0} \mid v_{0}\right)} d a_{0} d v_{0}\right)^{\frac{1}{2}} \Delta(a e)^{\frac{1}{2}(\mu+b)} .
\end{aligned}
$$

Thus, the following integral transform is well defined.

6.6. Definition. For any $f \in\left(\mathfrak{S F}_{\Gamma}\right)^{0}$, the Hankel transform $\mathcal{K}_{\pi}$ corresponding to the Bessel function $K_{\pi}$ is defined by

$$
\left(\mathcal{K}_{\pi} f\right)\left(a k\left(u_{0}\right)\right)=\int_{A K_{-\frac{1}{2}}} K_{\pi}\left(a_{0}^{*} a k\left(u_{0}\right) ; v_{0}\right) f\left(a_{0} k\left(v_{0}\right)\right) e^{-\left(v_{0} \mid v_{0}\right)} d a_{0} d v_{0} .
$$

6.7. Theorem. For $F \in \mathcal{H}_{\pi}$ denote

$$
F^{\#}(z, u)=\pi(z) \pi(k(u)) F\left(-z^{-1}, z^{-1} \circ u\right) .
$$

Then

(a) The mapping $\mathcal{U}: F \mapsto F^{\#}$ is a unitary operator on $\mathcal{H}_{\pi}$.

(b) The operator $\mathcal{K}_{\pi}$ on $\left(\mathfrak{S F}_{\Gamma}\right)^{0}$ extends uniquely to a unitary operator of $\mathfrak{S F}_{\Gamma}$.

(c) The unitary operator $\mathcal{T}$ given by (6.2.1) intertwines $\mathcal{K}_{\pi}$ and $\mathcal{U}$; i.e.,

$$
\mathcal{K}_{\pi}=\mathcal{T}^{-1} \mathcal{U} \mathcal{T}
$$

Proof. If $F \in \mathcal{H}_{\pi}$, then

$$
\begin{aligned}
& \int_{\Sigma}\left\|\pi\left(r(2 y-B(u, \bar{u}))^{\frac{1}{2}} k(-i u)\right)^{*^{-1}} F^{\#}(x+i y, u)\right\|^{2} \\
& \quad \times \Delta(2 y-B(u, \bar{u}))^{-2 \mu-b} d x d y d u \\
& =\int_{\Sigma}\left\|\pi\left(r(2 y-B(u, \bar{u}))^{\frac{1}{2}} k(-i u)\right)^{*^{-1}} \pi(z) \pi(k(u)) F\left(-z^{-1}, z^{-1} \circ u\right)\right\|^{2} \\
& \quad \times \Delta(2 y-B(u, \bar{u}))^{-2 \mu-b} d x d y d u \\
& =\int_{\Sigma} \operatorname{tr}\left\{F\left(-z^{-1}, z^{-1} \circ u\right)^{*} \pi(k(u))^{*} \pi(z)^{*} \pi(k(i u)) \pi(2 y-B(u, \bar{u}))^{-1}\right. \\
& \left.\times \pi(k(i u))^{*} \pi(z) \pi(k(u)) F\left(-z^{-1}, z^{-1} \circ u\right)\right\} \Delta(2 y-B(u, \bar{u}))^{-2 \mu-b} d x d y d u .
\end{aligned}
$$

Changing variables $z \mapsto-z^{-1}, u \mapsto-z^{-1} \circ u$ and applying Lemma 5.5, (3) is equal to

$$
\begin{aligned}
& \int_{\Sigma} \operatorname{tr}\left\{F(z, u)^{*} \pi\left(k(i u) r(2 y-B(u, \bar{u}))^{-1} k(i u)^{*}\right) F(z, u)\right\} \\
& \times \Delta(2 y-B(u, \bar{u}))^{-2 \mu-b} d x d y d u \\
& \quad=\|F\|^{2} .
\end{aligned}
$$

Thus, $F^{\#} \in \mathcal{H}_{\pi}$, and $\mathcal{U}$ is a unitary operator. (a) is proved. 
Let $f \in\left(\mathfrak{S} \mathfrak{F}_{\Gamma}\right)^{0}$ and set

$$
f^{\#}\left(a k\left(u_{0}\right)\right)=\left(\mathcal{K}_{\pi} f\right)\left(a k\left(u_{0}\right)\right) .
$$

It is clear that $f^{\#}$ satisfies the $\pi$-covariant condition (6.1.2).

We prove next that $f^{\#} \in \mathfrak{S F}_{\Gamma}$.

Formally constructing $\mathcal{T} f^{\#}$, we have

$$
\begin{aligned}
& \left(\mathcal{T} f^{\#}\right)(z, u) \\
& =\pi^{-\frac{n}{2}} \int_{A K_{-\frac{1}{2}}} e^{i(z \mid a e)} e^{\left(u \mid a u_{0}\right)} e^{-\left(u_{0} \mid u_{0}\right)} \pi\left(a k\left(i u_{0}\right)\right)^{*-1} f^{\#}\left(a k\left(u_{0}\right)\right) d a d u_{0} \\
& =\pi^{-\frac{n}{2}} \int_{A K_{-\frac{1}{2}}} e^{i(z \mid a e)} e^{\left(u \mid a u_{0}\right)} e^{-\left(u_{0} \mid u_{0}\right)} \pi\left(a k\left(i u_{0}\right)\right)^{*-1} \\
& \times\left(\int_{A K_{-\frac{1}{2}}} K_{\pi}\left(a_{0}^{*} a k\left(u_{0}\right) ; v_{0}\right) f\left(a_{0} k\left(v_{0}\right)\right) e^{-\left(v_{0} \mid v_{0}\right)} d a_{0} d v_{0}\right) d a d u_{0} \\
& =\pi^{-\frac{n}{2}} \int_{A K_{-\frac{1}{2}}}\left(\int_{A K_{-\frac{1}{2}}} e^{i(z \mid a e)} e^{\left(u \mid a u_{0}\right)} e^{-\left(u_{0} \mid u_{0}\right)} \pi\left(a k\left(i u_{0}\right)\right)^{*-1}\right. \\
& \left.\quad \times K_{\pi}\left(a_{0}^{*} a k\left(u_{0}\right) ; v_{0}\right) d a d u_{0}\right) f\left(a_{0} k\left(v_{0}\right)\right) e^{-\left(v_{0} \mid v_{0}\right)} d a_{0} d v_{0} .
\end{aligned}
$$

By (5.4.2), (4.8.2) and variable changes, we have

$$
\begin{aligned}
& \int_{A K_{-\frac{1}{2}}} e^{i(z \mid a e)} e^{\left(u \mid a u_{0}\right)} e^{-\left(u_{0} \mid u_{0}\right)} \pi\left(a k\left(i u_{0}\right)\right)^{*-1} K_{\pi}\left(a_{0}^{*} a k\left(u_{0}\right) ; v_{0}\right) d a d u_{0} \\
& =e^{-i\left(z^{-1} \mid a_{0} e\right)} e^{\left(z^{-1} \mid B\left(a_{0} v_{0}, \bar{u}\right)\right)} \pi(z) \pi\left(a_{0}^{*}\right)^{-1} \pi\left(k\left(a_{0}^{-1} u\right)\right) \pi\left(k\left(-i v_{0}\right)\right)^{*} \\
& =e^{-i\left(z^{-1} \mid a_{0} e\right)} e^{\left(z^{-1} \circ u \mid a_{0} v_{0}\right)} \pi(z) \pi(k(u)) \pi\left(a_{0}^{*}\right)^{-1} \pi\left(k\left(-i v_{0}\right)\right)^{*} .
\end{aligned}
$$

Thus,

$$
\begin{aligned}
& \left(\mathcal{T} f^{\#}\right)(z, u)=\pi^{-\frac{n}{2}} \int_{A K_{-\frac{1}{2}}} e^{-i\left(z^{-1} \mid a_{0} e\right)} e^{\left(z^{-1} \circ u \mid a_{0} v_{0}\right)} \\
& \times \pi(z) \pi(k(u)) \pi\left(a_{0} k\left(i v_{0}\right)\right)^{*-1} f\left(a_{0} k\left(v_{0}\right)\right) e^{-\left(v_{0} \mid v_{0}\right)} d a_{0} d v_{0} \\
& =\pi(z) \pi(k(u)) F\left(-z^{-1}, z^{-1} \circ u\right) ;
\end{aligned}
$$

i.e., formally

$$
F^{\#}=\mathcal{T} f^{\#} .
$$

Applying Corollary 4.9 with

$$
\phi\left(a k\left(u_{0}\right)\right)=f^{\#}\left(a k\left(u_{0}\right)\right) \Delta(a e)^{-\mu},
$$


we have

$$
\begin{aligned}
& \int_{A K_{-\frac{1}{2}}}\left(\Gamma_{\pi} f^{\#}\left(a k\left(u_{0}\right)\right) \mid f^{\#}\left(a k\left(u_{0}\right)\right)\right) e^{-\left(u_{0} \mid u_{0}\right)} d a d u_{0} \\
& =\int_{\Sigma}\left\|\pi\left(r(2 y-B(u, \bar{u}))^{\frac{1}{2}} k(-i u)\right)^{*^{-1}} F^{\#}(x+i y, u)\right\|^{2} \\
& \quad \times \Delta(2 y-B(u, \bar{u}))^{-2 \mu-b} d x d y d u \\
& =\int_{\Sigma}\left\|\pi\left(r(2 y-B(u, \bar{u}))^{\frac{1}{2}} k(-i u)\right)^{*^{-1}} F(x+i y, u)\right\|^{2} \\
& \quad \times \Delta(2 y-B(u, \bar{u}))^{-2 \mu-b} d x d y d u \\
& <\infty
\end{aligned}
$$

by (a). Thus, $f^{\#} \in \mathfrak{S F}_{\Gamma}$.

By (4) and (6),

$$
\mathcal{K}_{\pi} f=\mathcal{T}^{-1} \mathcal{U} \mathcal{T} f
$$

for all $f \in\left(\mathfrak{S} \mathfrak{F}_{\Gamma}\right)^{0}$. Hence $\mathcal{K}_{\pi}$ agrees with the unitary operator $\mathcal{T}^{-1} \mathcal{U} \mathcal{T}$ on a dense subspace and $\mathcal{K}_{\pi}$ extends uniquely from $\left(\mathfrak{S F}_{\Gamma}\right)^{0}$ to a unitary operator on $\mathfrak{S F}_{\Gamma}$. The theorem is proved.

We now study the reproducing kernel property of the space $\mathcal{H}_{\pi}$.

6.8. Reproducing kernels. By (6.2.3), $F=\mathcal{T} f$ satisfies that

$$
\begin{aligned}
|F(z, u)| & \leq\|f\|\left(\int_{A K_{-\frac{1}{2}}} e^{-2(t \mid a e)} e^{-\left(u_{0}-a^{*} u \mid u_{0}-a^{*} u\right)}\left\|\pi\left(a k\left(i u_{0}\right)\right)^{*-1}\right\|^{2} d a d u_{0}\right)^{\frac{1}{2}} \\
& \leq\|F\|\left(\int_{A K_{-\frac{1}{2}}} e^{-2(t \mid a e)} e^{-\left(u_{0}-a^{*} u \mid u_{0}-a^{*} u\right)}\left\|\pi\left(a k\left(i u_{0}\right)\right)^{*-1}\right\|^{2} d a d u_{0}\right)^{\frac{1}{2}}
\end{aligned}
$$

for $f \in \mathfrak{S F}_{\Gamma}$. Thus, for each $(z, u) \in \Sigma$ the point evaluation

$$
E_{(z, u)}: F \mapsto F(z, u)
$$

is a continuous linear operator from $\mathcal{H}_{\pi}$ to $\mathcal{V}_{\pi}$. Then by $[\mathrm{Ku}], \mathcal{H}_{\pi}$ is characterized by its reproducing kernel

$$
Q_{\pi}\left((z, u),\left(z^{\prime}, u^{\prime}\right)\right)=E_{(z, u)} E_{\left(z^{\prime}, u^{\prime}\right)}^{*}
$$

in the sense that

$$
\left(F\left(z^{\prime}, u^{\prime}\right) \mid v\right)=\left\langle F \mid Q_{\pi}\left(\cdot,\left(z^{\prime}, u^{\prime}\right)\right) v\right\rangle
$$

for all $F \in \mathcal{H}_{\pi}, v \in \mathcal{V}_{\pi}$ and $\left(z^{\prime}, u^{\prime}\right) \in \Sigma$.

We now obtain an integral representation for the reproducing kernel of the space $\mathcal{H}_{\pi}$.

6.9. Theorem. For each $\left(z^{\prime}, u^{\prime}\right) \in \Sigma$, the integral

$$
\begin{aligned}
& F_{\left(z^{\prime}, u^{\prime}\right)}(z, u) \\
& =\pi^{-n} \int_{A K_{-\frac{1}{2}}} e^{i\left(z-z^{\prime *} \mid a e\right)} e^{\left(u \mid a u_{0}\right)} e^{\left(a u_{0} \mid u^{\prime}\right)} e^{-\left(u_{0} \mid u_{0}\right)} \\
& \times \pi\left(a k\left(i u_{0}\right)\right)^{*-1} \Gamma_{\pi}^{-1} \pi\left(a k\left(i u_{0}\right)\right)^{-1} d a d u_{0}
\end{aligned}
$$


converges absolutely and defines a holomorphic function $F_{\left(z^{\prime}, u^{\prime}\right)}$ of $(z, u) . F_{\left(z^{\prime}, u^{\prime}\right)}$ is the reproducing kernel of $\mathcal{H}_{\pi}$ in the sense of (6.8.3); i.e.,

$$
\left(F\left(z^{\prime}, u^{\prime}\right) \mid v\right)=\left\langle F \mid F_{\left(z^{\prime}, u^{\prime}\right)}(\cdot) v\right\rangle
$$

for all $F \in \mathcal{H}_{\pi}$ and $v \in \mathcal{V}_{\pi}$.

Proof. Let $f \in \mathfrak{S F}_{\Gamma}$ such that $F=\mathcal{T} f$. Then by (6.2.1) and (6.1.4), we have

$$
\begin{aligned}
& \left(F\left(z^{\prime}, u^{\prime}\right) \mid v\right) \\
& =\left(\pi^{-\frac{n}{2}} \int_{A K_{-\frac{1}{2}}} e^{i\left(z^{\prime} \mid a e\right)} e^{\left(u^{\prime} \mid a u_{0}\right)} e^{-\left(u_{0} \mid u_{0}\right)} \pi\left(a k\left(i u_{0}\right)\right)^{*-1} f\left(a k\left(u_{0}\right)\right) d a d u_{0} \mid v\right) \\
& =\left\langle f \mid f_{\left(z^{\prime}, u^{\prime}\right)}(\cdot) v\right\rangle
\end{aligned}
$$

for $\left(z^{\prime}, u^{\prime}\right) \in \Sigma$ and $v \in \mathcal{V}_{\pi}$, where

$$
f_{\left(z^{\prime}, u^{\prime}\right)}\left(a k\left(u_{0}\right)\right)=\pi^{-\frac{n}{2}} e^{-i\left(a e \mid z^{\prime}\right)} e^{\left(a u_{0} \mid u^{\prime}\right)} \Gamma_{\pi}^{-1} \pi\left(a k\left(i u_{0}\right)\right)^{-1} .
$$

It is clear that the function $f_{\left(z^{\prime}, u^{\prime}\right)}$ satisfies (6.1.2). By Theorem 4.4, it satisfies (6.1.3). Thus, $f_{\left(z^{\prime}, u^{\prime}\right)}(\cdot) v \in \mathfrak{S F}_{\Gamma}$ for $v \in \mathcal{V}_{\pi}$.

Define

$$
F_{\left(z^{\prime}, u^{\prime}\right)} v=\mathcal{T} f_{\left(z^{\prime}, u^{\prime}\right)} v .
$$

for $v \in \mathcal{V}_{\pi}$. Then, $F_{\left(z^{\prime}, u^{\prime}\right)}$ can be expressed by (1) and

$$
\left(F\left(z^{\prime}, u^{\prime}\right) \mid v\right)=\left\langle f \mid f_{\left(z^{\prime}, u^{\prime}\right)}(\cdot) v\right\rangle=\left\langle F \mid F_{\left(z^{\prime}, u^{\prime}\right)}(\cdot) v\right\rangle
$$

for $v \in \mathcal{V}_{\pi}$. The theorem is proved.

\section{HOLOMORPHIC DISCRETE SERIES}

In this section, we construct equivalent irreducible unitary representations $S_{\pi}$ and $T_{\pi}$ of $G$ on the spaces $\mathfrak{S F}_{\Gamma}$ and $\mathcal{H}_{\pi}$, respectively, which are intertwined by the operator $\mathcal{T}$. We show that $S_{\pi}$ and $T_{\pi}$ are irreducible and square-integrable, and that they are in the holomorphic discrete series of $G$. We compute the reproducing kernel of $\mathcal{H}_{\pi}$ explicitly.

7.1. Definition. We define the $\mathcal{E}$-valued function $m_{\pi}$ by

$$
m_{\pi}(g,(z, u))=\pi(m(g,(z, u)))
$$

for $(g,(z, u)) \in G^{\mathbb{C}} \times\left(V_{2} \oplus V_{1}\right)$, where $m$ is given by (3.7.2). Then $m_{\pi}$ satisfies the multiplier identity (3.7.3).

For $F \in \mathcal{H}_{\pi}$ and $g \in G$, define

$$
\left(T_{\pi}(g) F\right)(z, u)=m_{\pi}(g,(z, u)) F\left(g^{-1} \cdot(z, u)\right)
$$

for $(z, u) \in \Sigma$.

7.2. Theorem. $T_{\pi}$ is a unitary representation of $G$ on $\mathcal{H}_{\pi}$. For generators a, $R\left(u_{0}\right), R\left(x_{0}\right)$, $j$ of $G$ given by (3.6.4), (3.6.6), (3.6.7) and (3.6.8), $T_{\pi}$ acts on $\mathcal{H}_{\pi}$ as follows:

(1)

$$
\left\{\begin{array}{l}
\left(T_{\pi}(a) F\right)(z, u)=\pi(a) F\left(a^{-1} z, a^{-1} u\right), \\
\left(T_{\pi}\left(R\left(u_{0}\right) F\right)(z, u)=\pi\left(k\left(-i u_{0}\right)\right)^{*} F\left(z-i B\left(u, \bar{u}_{0}\right)+\frac{i}{2} B\left(u_{0}, \bar{u}_{0}\right), u-u_{0}\right),\right. \\
\left(T_{\pi}\left(R\left(x_{0}\right) F\right)(z, u)=F\left(z-x_{0}, u\right)\right. \\
\left(T_{\pi}(j) F\right)(z, u)=\pi(z) \pi(k(u)) F\left(-z^{-1}, z^{-1} \circ u\right) .
\end{array}\right.
$$


Proof. Since $m_{\pi}$ satisfies the multiplier identity (3.7.3), $T_{\pi}$ is a representation. That is,

$$
T_{\pi}\left(g_{1} g_{2}\right) F=T_{\pi}\left(g_{1}\right) T_{\pi}\left(g_{2}\right) F
$$

for all $g_{1}, g_{2} \in G$ and $F \in \mathcal{H}_{\pi}$. Formula (1) follows from (7.1.2) directly. By the norm $(6.3 .1), T_{\pi}(a), T_{\pi}\left(R\left(u_{0}\right)\right), T_{\pi}\left(R\left(x_{0}\right)\right)$ are unitary operators on $\mathcal{H}_{\pi}$. That $T_{\pi}(j)$ is unitary follows from Theorem 6.7 .

7.3. Theorem. The following formulas define a unitary representation of $G$ on $\mathfrak{S} \mathfrak{F}_{\Gamma}$.

$$
\left\{\begin{array}{l}
\left(S_{\pi}\left(a_{0}\right) f\right)(a k(u))=f\left(a_{0}^{*} a k(u)\right) \\
\left(S_{\pi}\left(R\left(u_{0}\right)\right) f\right)(a k(u))=e^{-\frac{1}{2}\left(a^{*} u_{0} \mid a^{*} u_{0}\right)} e^{\left(u \mid a^{*} u_{0}\right)} f\left(a k\left(u-a^{*} u_{0}\right)\right) \\
\left(S_{\pi}\left(R\left(x_{0}\right)\right) f\right)(a k(u))=e^{-i\left(x_{0} \mid a e\right)} f(a k(u)) \\
\left(S_{\pi}(j) f\right)(a k(u))=\left(\mathcal{K}_{\pi} f\right)(a k(u))
\end{array}\right.
$$

Moreover, $S_{\pi}=\mathcal{T}^{-1} T_{\pi} \mathcal{T}$.

Proof. $S_{\pi}=\mathcal{T}^{-1} T_{\pi} \mathcal{T}$ follows directly from (1), (7.2.1), (6.2.1), (6.7.2) and a computation. Since $T_{\pi}$ is a unitary representation of $G$, so is $S_{\pi}$.

By [Di4, Theorem], we now show that the representation $T_{\pi}$ of $G$ is irreducible.

7.4. Theorem. The representation $T_{\pi}$ of $G$ on $\mathcal{H}_{\pi}$ is irreducible.

Proof. By Section 6.8, the point evaluation $E_{(z, u)}$ is a continuous operator from $\mathcal{H}_{\pi}$ to $\mathcal{V}_{\pi}$ for each $(z, u) \in \Sigma$.

Let $K$ be the maximal compact subgroup of $G^{\prime}$ and $c$ be the Cayley transform given by (2.7.3). Note that $K$ is the isotropy subgroup of 0 in $G^{\prime}$ and that $c \cdot 0=i e$. By (3.7.3), for $k \in K$ we have

$$
\begin{aligned}
m\left(c k c^{-1}, i e\right) & =m(c, i e) m\left(k c^{-1}, c^{-1} \cdot i e\right) \\
& =m(c, i e) m\left(k c^{-1}, 0\right) \\
& =m(c, i e) m(k, 0) m\left(c^{-1}, k^{-1} \cdot 0\right) \\
& =m(c, i e) k m\left(c^{-1}, 0\right) .
\end{aligned}
$$

Taking $k$ in (1) the identity element $I$ of $K$, we have

$$
m(c, i e) m\left(c^{-1}, 0\right)=m(I, i e)=I .
$$

Thus, $m(c, i e)^{-1}=m\left(c^{-1}, 0\right)$. Suppose that $l \in \mathcal{E}$ satisfies

$$
m_{\pi}\left(c k c^{-1}, i e\right) l m_{\pi}\left(c k c^{-1}, i e\right)^{*}=l
$$

for all $k \in K$. By (1) and (7.1.1), we have

$$
m_{\pi}(c, i e) \pi(k) m_{\pi}\left(c^{-1}, 0\right) l m_{\pi}\left(c^{-1}, 0\right)^{*} \pi\left(k^{*}\right) m_{\pi}(c, i e)^{*}=l,
$$

or

$$
\pi(k) m_{\pi}\left(c^{-1}, 0\right) l m_{\pi}\left(c^{-1}, 0\right)^{*} \pi\left(k^{*}\right)=m_{\pi}\left(c^{-1}, 0\right) l m_{\pi}\left(c^{-1}, 0\right)^{*} .
$$

Since $\pi$ is an irreducible representation of $K, m_{\pi}\left(c^{-1}, 0\right) l m_{\pi}\left(c^{-1}, 0\right) *$ is a constant. By the Theorem of [Di4], $T_{\pi}$ is irreducible.

7.5. Corollary. The representation $S_{\pi}$ of $G$ is irreducible.

We now compute the reproducing kernel $Q_{\pi}$ given by (6.8.2). 
7.6. Theorem. The reproducing kernel for $\mathcal{H}_{\pi}$ is given explicitly by the formula

$$
Q_{\pi}\left((z, u),\left(z^{\prime}, u^{\prime}\right)\right)=\gamma_{\pi} \pi\left(k\left(i u^{\prime}\right)^{*} r\left(z-z^{\prime *}\right) k(i u)\right),
$$

where $\gamma_{\pi}$ is a positive constant

$$
\gamma_{\pi}=\pi^{-n} d^{-1} \operatorname{tr}\left(\Gamma_{\pi}(0) \Gamma_{\pi}^{-1}\right),
$$

$d$ is the dimension of $\mathcal{V}_{\pi}$, and $\Gamma_{\pi}(0)$ and $\Gamma_{\pi}$ are given by (4.3.2) and (6.1.1), respectively.

Proof. Let $E_{(z, u)}$ be the point evaluation on $\mathcal{H}_{\pi}$, which is a continuous linear operator of $\mathcal{H}_{\pi}$ to $\mathcal{V}_{\pi}$ by Section 6.8. Rewrite (7.1.2) as

$$
E_{(z, u)} T_{\pi}(g)=m_{\pi}(g,(z, u)) E_{g^{-1} \cdot(z, u)}
$$

for $g \in G,(z, u) \in \Sigma$. Since $T_{\pi}$ is unitary,

$$
T_{\pi}(g)^{-1} E_{(z, u)}^{*}=E_{g^{-1} \cdot(z, u)}^{*} m_{\pi}(g,(z, u))^{*} .
$$

Thus,

$$
E_{\left(z^{\prime}, u^{\prime}\right)} E_{(z, u)}^{*}=m_{\pi}\left(g,\left(z^{\prime}, u^{\prime}\right)\right) E_{g^{-1} \cdot\left(z^{\prime}, u^{\prime}\right)} E_{g^{-1} \cdot(z, u)}^{*} m_{\pi}(g,(z, u))^{*}
$$

for $g \in G,(z, u),\left(z^{\prime}, u^{\prime}\right) \in \Sigma$.

Letting $(z, u)=\left(z^{\prime}, u^{\prime}\right)$ and $g^{-1} \cdot(z, u)=i e$ in (4), we have

$$
E_{(z, u)} E_{(z, u)}^{*}=m_{\pi}(g,(z, u)) E_{i e} E_{i e}^{*} m_{\pi}(g,(z, u))^{*} .
$$

Since $m_{\pi}$ satisfies the multiplier identity (3.7.3),

$$
\begin{aligned}
I=m_{\pi}\left(g g^{-1},(z, u)\right) & =m_{\pi}(g,(z, u)) m_{\pi}\left(g^{-1}, g^{-1} \cdot(z, u)\right) \\
& =m_{\pi}(g,(z, u)) m_{\pi}\left(g^{-1}, i e\right)
\end{aligned}
$$

and

$$
m_{\pi}(g,(z, u))=m_{\pi}\left(g^{-1}, i e\right)^{-1} .
$$

In particular, taking $g=R(x) R(u) r\left(t^{\frac{1}{2}}\right)$ with $(z, u)=\left(x+i\left(t+\frac{1}{2} B(u, \bar{u})\right), u\right)$, we have $g \cdot i e=(z, u)$. By (3.8.1)-(3.8.3), we have

$$
\begin{aligned}
m_{\pi}\left(g^{-1}, i e\right) & =m_{\pi}\left(r\left(t^{-\frac{1}{2}}\right) R(u)^{-1} R(-x), i e\right) \\
& =m_{\pi}\left(r\left(t^{-\frac{1}{2}}\right), i e\right) m_{\pi}\left(R(u)^{-1} R(-x), r\left(t^{\frac{1}{2}}\right) \cdot i e\right) \\
& =\pi\left(t^{-\frac{1}{2}}\right) m_{\pi}\left(R(u)^{-1}, i t\right) m_{\pi}(R(-x), R(u) \cdot i t) \\
& =\pi\left(t^{-\frac{1}{2}}\right) \pi(k(-i u))^{*} .
\end{aligned}
$$

By (5), (6) and (7),

$$
E_{(z, u)} E_{(z, u)}^{*}=\pi(k(i u))^{*} \pi\left(t^{\frac{1}{2}}\right) E_{i e} E_{i e}^{*} \pi\left(t^{\frac{1}{2}}\right) \pi(k(i u)) .
$$

Letting $(z, u)=\left(z^{\prime}, u^{\prime}\right)=i e$ and $g=c k c^{-1}$ in (4), where $k \in K$ and $c$ is the Cayley transform given by (2.7.3), we have

$$
E_{i e} E_{i e}^{*}=m_{\pi}\left(c k c^{-1}, i e\right) E_{i e} E_{i e}^{*} m_{\pi}\left(c k c^{-1}, i e\right)^{*} .
$$

Thus, $E_{i e} E_{i e}^{*}$ satisfies $(7.4 .2)$, and $m_{\pi}\left(c^{-1}, 0\right) E_{i e} E_{i e}^{*} m_{\pi}\left(c^{-1}, 0\right)^{*}$ is a constant $\gamma_{\pi}$. Substituting in (8), we have

$$
E_{(z, u)} E_{(z, u)}^{*}=\gamma_{\pi} \pi(k(i u))^{*} \pi\left(t^{\frac{1}{2}}\right) m_{\pi}\left(c^{-1}, 0\right)^{-1} m_{\pi}\left(c^{-1}, 0\right)^{*-1} \pi\left(t^{\frac{1}{2}}\right) \pi(k(i u)) .
$$


By $(2.7 .3),(3.7 .1),(3.7 .2)$ and $(7.1 .1), m_{\pi}\left(c^{-1}, 0\right)=\pi\left(\frac{\sqrt{2}}{2} e\right)$ and $m_{\pi}\left(c^{-1}, 0\right)^{-1}=$ $m_{\pi}\left(c^{-1}, 0\right)^{*^{-1}}=\pi(\sqrt{2} e)$. Thus

$$
\begin{aligned}
E_{(z, u)} E_{(z, u)}^{*} & =\gamma_{\pi} \pi(k(i u))^{*} \pi\left(\sqrt{2} t^{\frac{1}{2}}\right) \pi\left(\sqrt{2} t^{\frac{1}{2}}\right) \pi(k(i u)) \\
& =\gamma_{\pi} \pi(k(i u))^{*} \pi(2 t) \pi(k(i u)) .
\end{aligned}
$$

By (6.8.2), (6.9.1), (4.3.2) and calculating $E_{i e} E_{i e}^{*}$, we have (2). Now (1) follows from $[\mathrm{Ku}, \mathrm{Lemma}]$, and the theorem is proved.

We now study the square-integrability of $S_{\pi}\left(\right.$ and $\left.T_{\pi}\right)$.

7.7. Measure decomposition on $G$. Let $d x$ and $d u$ be Lebesgue measures on $J$ and $V_{1}$, respectively, and $d a$ Haar measure on $A . R\left(u_{0}\right)$ and $R\left(x_{0}\right)$ commute, and generate a subgroup $N$ of $G$. $A$ normalizes $N$, and $M=N A$ is a subgroup of $G$.

$$
\int_{M} f(m) d m=\int_{A} \int_{J \oplus V_{1}} f\left(R\left(x_{0}\right) R\left(u_{0}\right) a\right) d x_{0} d u_{0} d a
$$

defines a right Haar measure on $M$, and this equality is valid for any nonnegative function $f$. Moreover, $d_{l} m=d m^{-1}$ is a left Haar measure on $M$ and the Haar measure $d g$ on $G$ decomposes as $d g=d\left(m^{-1} j R\left(u_{0}\right) R\left(x_{0}\right)\right)=d m d x_{0} d u_{0}$. Thus the formula

$$
\int_{G} f(g) d g=\int_{M} \int_{J \oplus V_{1}} f\left(m^{-1} j R\left(x_{0}\right) R\left(u_{0}\right)\right) d m d x_{0} d u_{0}
$$

is valid for all nonnegative measurable functions $f$.

7.8. Theorem. For any $f_{1}$ and $f_{2}$ in $\mathfrak{S F}_{\Gamma}$,

$$
\begin{aligned}
& \int_{M}\left|\left\langle S_{\pi}(m) f_{1} \mid f_{2}\right\rangle\right|^{2} d m \\
& \leq c\left\|f_{1}\right\|^{2}\left(\int_{A K_{-\frac{1}{2}}}\left\|\Gamma_{\pi}^{\frac{1}{2}} f_{2}\left(a k\left(u_{0}\right)\right)\right\|^{2} e^{-\left(u_{0} \mid u_{0}\right)} \Delta(a e)^{-\mu-b} d a d u_{0}\right)
\end{aligned}
$$

for some constant $c$.

Proof. By (6.1.4) and (7.3.1),

$$
\begin{aligned}
& \left\langle S_{\pi}\left(R\left(u_{0}\right) R\left(x_{0}\right) a_{0}\right) f_{1} \mid f_{2}\right\rangle \\
& =\int_{A K_{-\frac{1}{2}}} e^{-i\left(x_{0} \mid a e\right)} e^{-\frac{1}{2}\left(a^{*} u_{0} \mid a^{*} u_{0}\right)} e^{\left(u \mid a^{*} u_{0}\right)} \\
& \times \operatorname{tr}\left\{f_{2}(a k(u))^{*} \Gamma_{\pi} f_{1}\left(a_{0}^{*} a k\left(u-a^{*} u_{0}\right)\right)\right\} e^{-(u \mid u)} d a d u .
\end{aligned}
$$


By (3.10.1), (3.10.2), (7.7.1) and the Plancherel formula for functions on $J$,

$$
\begin{aligned}
& \int_{M}\left|\left\langle S_{\pi}\left(R\left(u_{0}\right) R\left(x_{0}\right) a\right) f_{1} \mid f_{2}\right\rangle\right|^{2} d m \\
& =\int_{A} \int_{J \oplus V_{1}} \mid \int_{\Omega \oplus V_{1}} e^{-i\left(x_{0} \mid \xi\right)} e^{-\frac{1}{2}\left(\xi^{\frac{1}{2}} \circ u_{0} \mid \xi^{\frac{1}{2}} \circ u_{0}\right)} e^{\left(u \mid \xi^{\frac{1}{2}} \circ u_{0}\right)} \\
& \times\left.\operatorname{tr}\left\{f_{2}(\xi, u)^{*} \Gamma_{\pi} f_{1}\left(a_{0}^{*} \xi^{\frac{1}{2}}, u-\xi^{\frac{1}{2}} \circ u_{0}\right)\right\} e^{-(u \mid u)} \Delta(\xi)^{-\mu} d \xi d u\right|^{2} d x_{0} d u_{0} d a_{0} \\
& =(2 \pi)^{n_{1}} \int_{\left(\Omega \oplus V_{1}\right) \times A} \mid \int_{V_{1}} e^{-\frac{1}{2}\left(\xi \circ u_{0} \mid u_{0}\right)} e^{\left(u \mid \xi^{\frac{1}{2}} \circ u_{0}\right)} \\
& \times\left.\operatorname{tr}\left\{f_{2}(\xi, u)^{*} \Gamma_{\pi} f_{1}\left(a_{0}^{*} \xi^{\frac{1}{2}}, u-\xi^{\frac{1}{2}} \circ u_{0}\right)\right\} e^{-(u \mid u)} d u\right|^{2} \Delta(\xi)^{-2 \mu} d \xi d u_{0} d a_{0} \\
& =(2 \pi)^{n_{1}} \int_{A \times V_{1} \times A} \mid \int_{V_{1}} e^{-\frac{1}{2}\left(a^{*} u_{0} \mid a^{*} u_{0}\right)} e^{\left(u \mid a^{*} u_{0}\right)} \\
& \times\left.\operatorname{tr}\left\{f_{2}(a k(u))^{*} \Gamma_{\pi} f_{1}\left(a_{0}^{*} a k\left(u-a^{*} u_{0}\right)\right)\right\} e^{-(u \mid u)} d u\right|^{2} \Delta(a e)^{-\mu} d a_{0} d u_{0} d a .
\end{aligned}
$$

By [Di2, Theorem 4.3] with the normalized measure $\pi^{-r b} d u_{0}$ and variable changes $a^{*} u_{0} \mapsto u_{0}, a_{0}^{*} a \mapsto a_{0}$,

$$
\begin{aligned}
& \int_{M}\left|\left\langle S_{\pi}\left(R\left(u_{0}\right) R\left(x_{0}\right) a_{0}\right) f_{1} \mid f_{2}\right\rangle\right|^{2} d m \\
& \leq(2 \pi)^{n_{1}} \pi^{r b} \int_{A \times V_{1} \times A} \operatorname{tr}\left\{\left(\Gamma_{\pi}^{\frac{1}{2}} f_{2}\left(a k\left(u_{0}\right)\right)\right)^{*}\left(\int_{V_{1}}\left\|\Gamma_{\pi}^{\frac{1}{2}} f_{1}\left(a_{0}^{*} a k(u)\right)\right\|^{2} e^{-(u \mid u)} d u\right)\right. \\
& \left.\quad \times \Gamma_{\pi}^{\frac{1}{2}} f_{2}\left(a k\left(u_{0}\right)\right)\right\} e^{-\left(u_{0} \mid u_{0}\right)} \Delta(a e)^{-\mu-b} d a_{0} d u_{0} d a \\
& =(2 \pi)^{n_{1}} \pi^{r b}\left\|f_{1}\right\|^{2} \int_{A K_{-\frac{1}{2}}}\left\|\Gamma_{\pi}^{\frac{1}{2}} f_{2}\left(a_{0} k\left(u_{0}\right)\right)\right\|^{2} e^{-\left(u_{0} \mid u_{0}\right)} \Delta\left(a_{0} e\right)^{-\mu-b} d a_{0} d u_{0} .
\end{aligned}
$$

The theorem is proved.

7.9. Theorem. For any $f_{1}$ and $f_{2}$ in $\mathfrak{S F}_{\Gamma}$,

$$
\begin{aligned}
& \int_{G}\left|\left\langle S_{\pi}(g) f_{1} \mid f_{2}\right\rangle\right|^{2} d g \\
& \leq c\left\|f_{1}\right\|^{2}\left\|f_{2}\right\|^{2} \int_{\Omega \oplus V_{1} \oplus V_{1}}\left(\Gamma_{\pi} K_{\pi}\left(\xi, u ; v_{0}\right) \mid K_{\pi}\left(\xi, u ; v_{0}\right)\right) \\
& \times \Delta(\xi)^{-2 \mu-b} e^{-(u \mid u)} e^{-\left(v_{0} \mid v_{0}\right)} d \xi d u d v_{0}
\end{aligned}
$$

for some constant $c$. 
Proof. By (7.7.2) and (7.8.2),

$$
\begin{aligned}
& \int_{G}\left|\left\langle S_{\pi}(g) f_{1} \mid f_{2}\right\rangle\right|^{2} d g \\
& =\int_{J \oplus V_{1}} \int_{M}\left|\left\langle S_{\pi}(m) f_{2} \mid S_{\pi}\left(j R\left(u_{0}\right) R\left(x_{0}\right)\right) f_{1}\right\rangle\right|^{2} d m d x_{0} d u_{0} \\
& \leq\left. 2^{n_{1}} \pi^{n}|| f_{2}\right|^{2} \int_{J \oplus V_{1}}\left(\left.\int_{A K_{-\frac{1}{2}}}|| \Gamma_{\pi}^{\frac{1}{2}} S_{\pi}\left(j R\left(u_{0}\right) R\left(x_{0}\right)\right) f_{1}(a k(u))\right|^{2}\right. \\
& \left.\quad \times e^{-(u \mid u)} \Delta(a e)^{-\mu-b} d a d u\right) d x_{0} d u_{0} .
\end{aligned}
$$

We assume temporarily that $f_{1}$ lies in the dense subspace $\left(\mathfrak{S F}_{\Gamma}\right)^{0}$ of $\mathfrak{S F}_{\Gamma}$ for which $\mathcal{K}_{\pi}$ is given by (6.6.1). Then

$$
\begin{aligned}
& \left(S_{\pi}\left(j R\left(u_{0}\right) R\left(x_{0}\right)\right) f_{1}\right)(a k(u)) \\
& =\int_{A K_{-\frac{1}{2}}} K_{\pi}\left(a_{0} a^{*} k(u) ; v_{0}\right) e^{-i\left(x_{0} \mid a_{0} e\right)} e^{-\frac{1}{2}\left(a_{0}^{*} u_{0} \mid a_{0}^{*} u_{0}\right)} \\
& \quad \times e^{\left(v_{0} \mid a_{0}^{*} u_{0}\right)} f_{1}\left(a_{0} k\left(v_{0}-a_{0}^{*} u_{0}\right)\right) d a_{0} d v_{0} .
\end{aligned}
$$

By (2), (3), the Plancherel formula, and [Di2, Theorem 4.3],

$$
\begin{aligned}
& \int_{G}\left|\left\langle S_{\pi}(g) f_{1} \mid f_{2}\right\rangle\right|^{2} d g \\
& \leq c|| f_{1}\left\|^{2}\right\| f_{2} \|^{2} \int_{\Omega \oplus V_{1} \oplus V_{1}}\left(\Gamma_{\pi} K_{\pi}\left(\xi, u ; v_{0}\right) \mid K_{\pi}\left(\xi, u ; v_{0}\right)\right) \\
& \quad \times \Delta(\xi)^{-2 \mu-b} e^{-(u \mid u)} e^{-\left(v_{0} \mid v_{0}\right)} d \xi d u d v_{0} .
\end{aligned}
$$

This proves (1) on a dense subset $\left(\mathfrak{S F}_{\Gamma}\right)^{0}$ of $\mathfrak{S F}_{\Gamma}$, but it follows readily from Fatou's lemma that (1) holds for all $f_{1}, f_{2} \in \mathfrak{S F}_{\Gamma}$, which completes the proof of the theorem.

7.10. Holomorphic discrete series. By Corollary 7.5 and Theorem $7.9, S_{\pi}$ (also $T_{\pi}$ ) are in the holomorphic discrete series of $G$. As shown in [RV1], the representations $T_{\pi}$ constitute the holomorphic discrete series of representations of $G$. The condition $\omega(\pi)>2 \mu+b-1$ (cf. (5.8.1)) is equivalent to Harish-Chandra's condition on the highest weight of $\pi$ for the existence of the holomorphic discrete series.

\section{REFERENCES}

[B1] S. Bochner, Bessel functions and modular relations of higher type and hyperbolic differential equations, Comm. Sem. Math. Univ. Lund. (1952), 12-20. MR 15:422f

[B2] Lectures on Fourier integrals, Ann. Math. Stud. 42, Princeton, 1959. MR 21:5851

[Dib] H. Dib, Fonctions de Bessel Sur une algebre de Jordan, J. Math. Pures Appl. 69 (1990), 403-448. MR 92e:33017

[Di1] H. Ding, Operator-valued Bessel functions on Schrödinger-Fock spaces and Siegel domains of type II, J. Func. Anal. 106 (1992), 189-225. MR 93f:32034

[Di2] Operator-valued Bessel functions, holomorphic discrete series and harmonic representations of $U(p, q)$, J. Func. Anal. 108 (1992), 187-230. MR 93m:22014

[Di3] , Bessel functions on quaternionic Siegel domains, J. Func. Anal. 120 (1994), 1-47. MR 95g:22022

[Di4] - Hilbert spaces of vector-valued holomorphic functions and irreducibility of multiplier representations, Contemp. Math. 138 (1992), 119-122. MR 94c:22010 
[DG1] H. Ding and K. I. Gross, Operator-valued Bessel functions on Jordan algebras, J. Reine Angew. Math. 435 (1993), 157-196. MR 93m:33010

[DG2] - Operator-valued Bessel functions on Jordan algebras, II: Analytic continuation, in preparation.

[Do1] J. Dorfmeister, Quasisymmetric Siegel domains and the automorphisms of homogeneous Siegel domains, Amer. J. Math. 102 (1980), 537-563. MR 81g:32028

[Do2] - Homogeneous Siegel domains, Nagoya Math. J. 86 (1982), 39-83. MR 84b:32042

[EHW] T. Enright, R. Howe and N. Wallach, A classification of unitary highest weight modules, Proceedings, Representation Theory of Reductive Groups, 1982, pp. 97-149. MR 86c:22028

[FK1] J. Faraut and A. Koranyi, Function spaces and reproducing kernels on bounded symmetric domains, J. Func. Anal. 88 (1990), 64-89. MR 90m:32049

[FK2] - Analysis on symmetric cones, Oxford Sci. Publ., 1994. MR 98g:17031

[Gi] S. G. Gindikin, Analysis on homogeneous domains, Russian Math. Surveys 19 (1964), $1-90$.

[GR] K. I. Gross and D. St. P. Richards, Special functions of matrix argument I: Algebraic induction, zonal polynomials, and hypergeometric functions, Trans. Amer. Math. Soc. 301 (1987), 781-811. MR 88m:22018

[GK1] K. I. Gross and R. A. Kunze, Bessel functions and representation theory, I, J. Func. Anal. 22 (1976), 73-105. MR 54:3305

[GK2] - Bessel functions and representation theory, II, holomorphic discrete series and metaplectic representations, J. Func. Anal. 25 (1977), 1-49. MR 56:12181

$[\mathrm{H}] \quad$ S. Helgason, Differential geometry, Lie groups, and symmetric spaces, Academic Press, 1978. MR 80k:53081

[Her] C. Herz, Bessel functions of matrix argument, Ann. Math. 61 (1955), 474-523. MR 16:1107e

[Ho1] R. Howe, Remarks on classical invariant theory, Trans. Amer. Math. Soc. 313 (1989), 539-570. MR 90h:22015a

[Ho2] - Transcending classical invariant theory, J. Amer. Math. Soc. 2 (1989), 535-552. MR 90k:22016

[J] A. T. James, Distributions of matrix variates and latent roots derived from normal samples, Ann. Math. Stat. 35 (1964), 475-501. MR 31:5286

[Kan] S. Kaneyuki, Homogeneous bounded domains and Siegel domains, Lecture notes, SpringerVerlag, 1971. MR 49:3231

[KV] M. Kashiwara and M. Vergne, On the Segal-Shale-Weil representations and harmonic polynomials, Invent. Math. 44 (1978), 1-47. MR 57:3311

[Kn] A. W. Knapp, Representation theory of semisimple Lie groups, an overview based on examples, Princeton, 1986. MR 87j:22022

[Koe] M. Koecher, Jordan algebras and their applications, Lecture notes, Univ. of Minn., 1962.

$[\mathrm{Ku}] \quad$ R. A. Kunze, On the irreducibility of certain multiplier representations, Bull. Amer. Math. Soc. 68 (1962), 93-94. MR 24:A3239

[L1] O. Loos, Bounded symmetric domains and Jordan pairs, Lecture notes, Univ. of California, Irvine, 1977.

[L2] _ Jordan pairs, Lecture notes, Springer-Verlag, 1975. MR 56:3071

[L3] — On algebraic groups defined by Jordan pairs, Nagoya Math. J. 74 (1979), 23-66. MR 80i: 17030

[M] S. Murakami, On automorphisms of Siegel domains, Lecture notes in mathematics 286, Springer-Verlag, 1971. MR 51:944

[P] I. I. Pyatetskii-Shapiro, Automorphic functions and the geometry of classical domains, Fizmatgiz, New York, 1961; English transl., New York, 1969.

[RV1] H. Rossi and M. Vergne, Representations of certain solvable Lie groups on Hilbert spaces of holomorphic functions and the application to the holomorphic discrete series of a semisimple Lie group, J. Func. Anal. 13 (1973), 324-389. MR 53:10989

[RV2] _ Analytic continuation of the holomorphic discrete series of a semisimple Lie group, Acta Math. 136 (1976), 1-59. MR 58:1032

[Se] I. E. Segal, Transforms for operators and symmetric automorphisms over a locally compact abelian group, Math. Scand. 13 (1963), 31-43. MR 29:486 
[Sh] D. Shale, Linear symmetries of free Boson fields, Trans. Amer. Math. Soc. 103 (1962), 149-169. MR 25:956

[W1] N. Wallach, The analytic continuation of the discrete series, I, Trans. Amer. Math. Soc. 251 (1979), 1-17. MR 81a:22009

[W2] The analytic continuation of the discrete series, II, Trans. Amer. Math. Soc. 251 (1979), 18-37. MR 81a:22009

[We] A. Weil, Sur certaines groupes d'operateurs unitaires, Acta Math. 111 (1964), 143-211. MR 29:2439

Department of Mathematics and Computer Science, St. Louis University, St. Louis, MissOuRI 63103

E-mail address: dingh@sluvca.slu.edu 